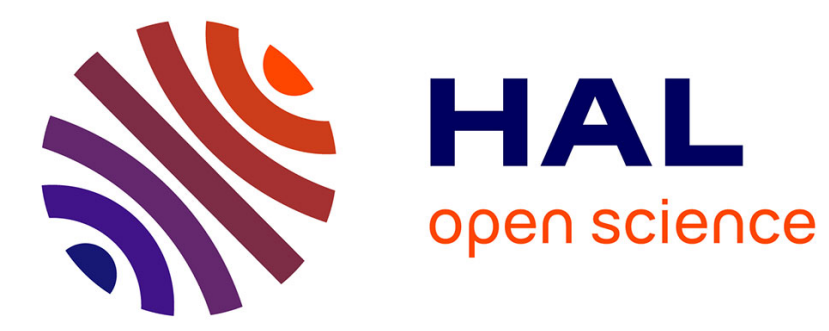

\title{
Indifference fee rate for variable annuities
}

Etienne Chevalier, Thomas Lim, Ricardo Romo Roméro

\section{To cite this version:}

Etienne Chevalier, Thomas Lim, Ricardo Romo Roméro. Indifference fee rate for variable annuities. 2014. hal-01017157

\section{HAL Id: hal-01017157 https://hal.science/hal-01017157}

Preprint submitted on 2 Jul 2014

HAL is a multi-disciplinary open access archive for the deposit and dissemination of scientific research documents, whether they are published or not. The documents may come from teaching and research institutions in France or abroad, or from public or private research centers.
L'archive ouverte pluridisciplinaire HAL, est destinée au dépôt et à la diffusion de documents scientifiques de niveau recherche, publiés ou non, émanant des établissements d'enseignement et de recherche français ou étrangers, des laboratoires publics ou privés. 


\title{
Indifference fee rate for variable annuities
}

\author{
Etienne CHEVALIER * Thomas LIM $^{\dagger}$ Ricardo ROMO ROMERO $\ddagger$
}

\begin{abstract}
In this paper, we work on indifference valuation of variable annuities and give a computation method for indifference fees. We focus on the guaranteed minimum death benefits and the guaranteed minimum living benefits and allow the policyholder to make withdrawals. We assume that the fees are continuously payed and that the fee rate is fixed at the beginning of the contract. Following indifference pricing theory, we define indifference fee rate for the insurer as a solution of an equation involving two stochastic control problems. Relating these problems to backward stochastic differential equations with jumps, we provide a verification theorem and give the optimal strategies associated to our control problems. From these, we derive a computation method to get indifference fee rates. We conclude our work with numerical illustrations of indifference fees sensibilities with respect to parameters.
\end{abstract}

Keywords: Variable annuities, indifference pricing, stochastic control, utility maximization, backward stochastic differential equation.

MSC2000 subject classification: 60H99, 91B30, 93E20.

\footnotetext{
${ }^{*}$ Universitï $₫ \frac{1}{2}$ d'Evry Val d'Essonne, Laboratoire de Mathï $₫ \frac{1}{2}$ matiques et Modï $\frac{1}{2}$ lisation d'Evry, CNRS UMR 8071, France, etienne.chevalier@univ-evry.fr.

${ }^{\dagger}$ ENSIIE, Laboratoire de Mathï $₫ \frac{1}{2}$ matiques et Modï $\frac{1}{2}$ lisation d'Evry, CNRS UMR 8071, France, thomas.lim@ensiie.fr.

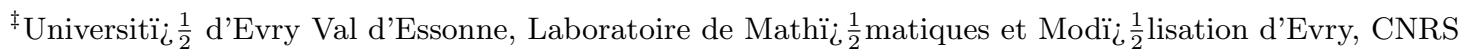
UMR 8071, France, ricardo.romo@univ-evry.fr.
} 


\section{Introduction}

Introduced in the 1970s in the United States (see [22]), variable annuities are equity-linked contracts between a policyholder and an insurance company. The policyholder gives an initial amount of money to the insurer. This amount is then invested in a reference portfolio until a preset date, until the policyholder withdraws from the contract or until he dies. At the end of the contract, the insurance pays to the policyholder or to his dependents a pay-off depending on the performance of the reference portfolio. In the 1990s, insurers included put-like derivatives which provided some guarantees to the policyholder. The most usual are guaranteed minimum death benefits (GMDB) and guaranteed minimum living benefits (GMLB). For a GMDB (resp. GMLB) contract, if the insured dies before the contract maturity (resp. is still alive at the maturity) he or his dependents obtain a benefit corresponding to the maximum of the current account value and of a guaranteed benefit. There exist various ways to fix this guaranteed benefit and we refer to [2] for more details. These products mainly present three risks for the insurer. First, as the insurer offers a putlike derivative on a reference portfolio to the client, he is considerably exposed to market risk. Moreover, variable annuity policies could have very long maturities so the pricing and hedging errors due to the model choice for the dynamic of the reference portfolio and the interest rates could be very important. The second risk faced by the insurer is the death of his client, this leads to the formulation of a problem with random maturity. Finally, the client may decide at any moment to withdraw, totally or partially, from the contract. Throughout the paper we shall assume that there is a rate of partial withdrawal that could be stochastic or not but we do not assume that it results from an optimal strategy of the insured. In case of total withdrawal, the insured may pay some penalties and will receive the maximum of the account facial value and of a guaranteed benefit minus the amount of previous partial withdrawals.

With the commercial success of variable annuities, the pricing and hedging of these products have been studied in a growing literature. Following the pioneering work of Boyle and Schwartz (see [8]), non-arbitrage models allow to extend the Black-Scholes framework to insurance issues. Milvesky and Posner (see [18]) are, up to our knowledge, the first to apply risk neutral option pricing theory to value GMDB in variable annuities. Withdrawal options are studied in [10] and [21], and a general framework to define variable annuities is presented in [2]. Milevsky and Salisbury (see [19]) focus on the links between American put options and dynamic optimal withdrawal policies. This problem is studied in [1] where an Hamilton-Jacobi-Bellman (HJB) equation is derived for a singular control problem where the control is the continuous withdrawal rate. The GMDB pricing problem is described as an impulse control problem in [3]. The authors model the GMDB problem as a stochastic control problem, derive an HJB equation and solve it numerically. The assumptions needed to get these formulations are the Markovianity of the stochastic processes involved and the existence of a risk neutral probability. The variable annuity policies with GMDB and GMLB are long term products therefore models for assets and interest rates have to be as rich as possible. Moreover, as we obviously face an incomplete market model, the price obtained strongly depends on the arbitrary choice of a risk neutral probability. 
This paper attempts to get an answer to these issues. We shall not make restrictive assumptions on the reference portfolio and the interest rate dynamics. As a result, our problem is not Markovian and we will not be able to derive HJB equations to characterize our value functions. We overcome this difficulty thanks to backward stochastic differential equations (BSDEs) following ideas from [12, [15] and [20]. In our case, we have to solve BSDE with random terminal time. For that we apply very recent results on BSDEs with jump (see for example [1] and [17]). Moreover, we shall not use non-arbitrage arguments to price and hedge variable annuity policies. We will assume that the fees, characterized by a preset fee rate, are continuously taken by the insurer from the policyholder's account and we will define an indifference fee rate for the insurer. Indifference pricing is a standard approach in mathematical finance to determine the price of a contingent claim in an incomplete market. This is a utility-based approach that can be summarized as follows. On the one hand, the investor may maximize his expected utility under optimal trading, investing only in the financial market. On the other hand, he could sell the contingent claim, optimally invest in the financial market and make a pay-off at the terminal time. The indifference price of this contingent claim is then the price such that the insurer gets the same expected utility in each case. For more details, we refer to the monograph of Carmona (see [9]).

The paper is organized as follows. In Section 1, we define the market model, the random times of death and total withdraw, then variable annuities with GMDB and/or GMLB are defined. We recall the main examples of guarantees associated to. Section 2 is devoted to indifference fee rates. They are defined as solutions of an equality between two regular stochastic control problems. These consist in maximizing the expected utility of the terminal wealth of the insurer portfolio in two cases: when the insurer has not sold variable annuities and when he has. Value functions of these two problems are respectively characterized as initial values of BSDEs. This characterization is well known for the first problem (see [15] and [20]) but demands to solve some technical issues for the second one. We conclude this section with a rigorous study of the existence of indifference fee rates in the usual cases i.e. with roll-up or ratchet guarantee. Finally, in Section 3, we conclude our paper with numerical illustrations of sensibilities of indifference fees with respect to model and market parameters.

\section{Model for variable annuities}

This section is divided as follows, Subsection 1.1 introduces the model for the underlying financial market in which the insurer invests. Subsection 1.2 describes the terminal date of a variable annuity policy. This one may be due to a total withdraw or to the death of the insured. The variable annuity products and the dynamics of the different processes that deal with are introduced in Subsection 1.3 .

\subsection{The financial market model}

Let $(\Omega, \mathcal{G}, \mathbb{P})$ be a complete probability space. We assume that this space is equipped with a one-dimensional standard Brownian motion $B$ and we denote by $\mathbb{F}:=\left(\mathcal{F}_{t}\right)_{t \geq 0}$ the right 
continuous complete filtration generated by $B$.

We consider a financial market on the time interval $[0, T]$ where $T>0$ corresponds to the expiration date of the variable annuities studied. We suppose that the financial market is composed by a riskless bond with an interest rate $r$ and a reference portfolio of risky assets underlying the variable annuity policy. The price processes $\hat{S}^{0}$ of the riskless bond and $\hat{S}$ of a share of the underlying risky portfolio are assumed to be solution of the following linear stochastic differential equations

$$
\begin{aligned}
d \hat{S}_{t}^{0} & =r_{t} \hat{S}_{t}^{0} d t, \quad \forall t \in[0, T], \quad \hat{S}_{0}^{0}=1, \\
d \hat{S}_{t} & =\hat{S}_{t}\left(\mu_{t} d t+\sigma_{t} d B_{t}\right), \quad \forall t \in[0, T], \quad \hat{S}_{0}=s>0,
\end{aligned}
$$

where $\mu, \sigma$ and $r$ are $\mathbb{F}$-adapted processes satisfying the following assumptions.

\section{Assumption A1.}

(i) The processes $\mu, \sigma$ and $r$ are $\mathbb{P}-$ a.s. bounded.

(ii) The process $\sigma$ is $\mathbb{P}-$ a.s. lower bounded by a positive constant $\underline{\sigma}$.

We shall denote by $S_{t}$ the discounted value of $\hat{S}_{t}$ at time $t \in[0, T]$, i.e. $S_{t}:=e^{-\int_{0}^{t} r_{s} d s} \hat{S}_{t}$.

The insurer invests on this financial market. For $t \in[0, T]$, we denote by $\pi_{t}^{0}$ (resp. $\pi_{t}$ ) the discounted amount of money invested in the riskless bond (resp. the risky portfolio). We suppose that the process $\pi$ is $\mathbb{F}$-adapted and satisfies the following integrability condition

$$
\int_{0}^{T}\left|\pi_{s} \mu_{s}\right| d s+\int_{0}^{T}\left|\pi_{s} \sigma_{s}\right|^{2} d s<+\infty, \quad \mathbb{P}-\text { a.s. }
$$

Assuming that the strategy of the insurer is self-financed and denoting by $X_{t}^{x, \pi}$ the discounted value of the insurer portfolio at time $t$ with initial capital $x \in \mathbb{R}^{+}$and following the strategy $\pi$, we have

$$
X_{t}^{x, \pi}=x+\int_{0}^{t} \pi_{s}\left(\mu_{s}-r_{s}\right) d s+\int_{0}^{t} \pi_{s} \sigma_{s} d B_{s}, \quad \forall t \in[0, T] .
$$

If the initial capital is null we denote $X_{t}^{\pi}$ the wealth instead of $X_{t}^{0, \pi}$.

We consider that the insurer wants to maximize the expected value of the utility of his terminal wealth $U\left(X_{T}^{x, \pi}\right)$ on an admissible strategies set, where $U(x):=-\exp (-\gamma x)$ with $\gamma>0$. Both theory and pratice have shown that it is appropriate to use exponantial utility functions. Since the decisions do not depend on the initial wealth of the insurer, it is well adapted to our problem of pricing one set of policies. Moreover an appealing feature of decision making using exponantial utility function is that decisions are based on comparisons between moment generating functions. They capture all the characteristics of the random outcomes being compared, so that comparisons are based on a wide range of features. We refer to [5] for more details about this choice.

In the following definition, we define the set of admissible strategies for the insurer, 
making usual restrictions that ensure some integrability properties for the processes involved.

Definition 1.1. ( $\mathbb{F}$-admissible strategy). For any $0 \leq u \leq v \leq T$, the set of admissible trading strategies $\mathcal{A}^{\mathbb{F}}[u, v]$ consists of all $\mathbb{F}$-adapted processes $\pi=\left(\pi_{t}\right)_{u \leq t \leq v}$ which satisfy

$$
\mathbb{E}\left[\int_{u}^{v}\left|\pi_{t} \sigma_{t}\right|^{2} d t\right]<\infty
$$

and

$$
\left\{\exp \left(-\gamma X_{\theta}^{x, \pi}\right), \theta \text { is an } \mathbb{F} \text {-stopping time with values in }[u, v]\right\}
$$

is uniformly integrable.

\subsection{Exit time of a variable annuity policy}

We consider two random times $\theta^{d}$ and $\theta^{w}$ which respectively represent the death time of the insured and the time of early closure of the insured account. We denote by $\tau=\theta^{d} \wedge \theta^{w}$. The random time $\tau$ is not assumed to be an $\mathbb{F}$-stopping time. We therefore use in the sequel the standard approach of filtration enlargement by considering $\mathbb{G}$ the smallest right continuous extension of $\mathbb{F}$ that turns $\tau$ into a $\mathbb{G}$-stopping time (see e.g. [4, 17]). More precisely $\mathbb{G}:=\left(\mathcal{G}_{t}\right)_{t \geq 0}$ is defined by

$$
\mathcal{G}_{t}:=\bigcap_{\varepsilon>0} \tilde{\mathcal{G}}_{t+\varepsilon}
$$

for all $t \geq 0$, where $\tilde{\mathcal{G}}_{s}:=\mathcal{F}_{s} \vee \sigma\left(\mathbb{1}_{\tau \leq u}, u \in[0, s]\right)$, for all $s \geq 0$.

We impose the following assumptions, which are usual in filtration enlargement theory.

Assumption A2. (H-hypothesis) The process $B$ remains a $\mathbb{G}$-Brownian motion.

The interpretation of the $\mathrm{H}$-hypothesis is an assymetric dependance structure between $B$ and $\tau$. From a financial point of view, it means that the exit time $\tau$ may depend on the financial market randomness represented by $B$. On the contrary, the financial market does not depend on $\tau$.

In the sequel, we introduce the process $N$ defined by $N=\left(1_{\{\tau \leq t\}}\right)_{0 \leq t \leq T}$.

Assumption A3. The process $N$ admits an $\mathbb{F}$-compensator of the form $\int_{0}^{\wedge} \lambda_{t} d t$, i.e. $N-\int_{0}^{. \wedge \tau} \lambda_{t} d t$ is a $\mathbb{G}$-martingale, where $\lambda$ is a bounded $\mathbb{F}$-adapted process.

$M$ denotes the $\mathbb{G}$-martingale defined by $M_{t}:=N_{t}-\int_{0}^{t \wedge \tau} \lambda_{s} d s$, for all $t \geq 0$.

If the investment strategy of the insurer depends on this exit time, we shall enlarge the set of admissible strategies through the following definition.

Definition 1.2. (G-admissible strategy). For any $0 \leq u \leq v \leq T$, the set of admissible 
trading strategies $\mathcal{A}^{\mathbb{G}}[u, v]$ consists of all $\mathbb{G}$-predictable processes $\pi=\left(\pi_{t}\right)_{u \leq t \leq v}$ which satisfy

$$
\mathbb{E}\left[\int_{u}^{v}\left|\pi_{t} \sigma_{t}\right|^{2} d t\right]<\infty
$$

and

$$
\left\{\exp \left(-\gamma X_{\theta}^{x, \pi}\right), \theta \text { is a } \mathbb{G} \text {-stopping time with values in }[u, v]\right\}
$$

is uniformly integrable.

\subsection{Variable annuity policy}

Let $\mathbb{T}:=\left(t_{i}\right)_{0 \leq i \leq n}$ be the set of policy anniversary dates, with $t_{0}=0$ and $t_{n}=T$. We also denote $t_{n+1}=+\infty$.

The first process to consider is the discounted account value $A^{p}$. The dynamic of the process $A^{p}$ is as follow

$$
d A_{t}^{p}=A_{t}^{p}\left[\left(\mu_{t}-r_{t}-\xi_{t}-p\right) d t+\sigma_{t} d B_{t}\right], \quad \forall t \in[0, T]
$$

with initial value $A_{0}, p$ is the fee rate taken by the insurer from the account of the insured and the process $\xi$ is a $\mathbb{G}$-predictable, non-negative and bounded process. $\xi_{t}$ represents the withdrawal rate chosen by the insured at time $t \in[0, T]$. We emphasize that $\xi$ is not necessarily a process resulting from an optimal control of the insured as, for example, in [3], [11] and [19].

For any $\pi \in \mathcal{A}^{\mathbb{G}}[0, T]$, we extend the definition of the process $X^{x, \pi}$ where $X_{t}^{x, \pi}$ is the discounted wealth of the portfolio invested in the financial market at time $t \in[0, T]$ and we set

$$
d X_{t}^{x, \pi}=\pi_{t}\left(\mu_{t}-r_{t}\right) d t+\pi_{t} \sigma_{t} d B_{t}, \quad \forall t \in[0, T]
$$

with $X_{0}^{x, \pi}=x$.

The second quantity to define is the pay-off of the variable annuities. Let $p \geq 0$, the pay-off is paid at time $T \wedge \tau$ to the insured or his dependents and is equal to the following random variable

$$
\hat{F}(p):=\hat{F}_{T}^{L}(p) \mathbb{1}_{\{T<\tau\}}+\hat{F}_{\tau}^{D}(p) \mathbb{1}_{\left\{\tau=\theta^{d} \leq T\right\}}+\hat{F}_{\tau}^{W}(p) \mathbb{1}_{\left\{\tau=\theta^{w}<\theta^{d} ; \tau \leq T\right\}} .
$$

$\hat{F}_{T}^{L}(p)$ is the pay-off if the policyholder is alive at time $T$ and has not totally withdrawn his money from his account. $\hat{F}_{\tau}^{D}(p)$ is the pay-off if the policyholder is dead at time $\tau . \hat{F}_{\tau}^{W}(p)$ is the pay-off if the policyholder totally withdraws his money from his account at time $\tau$. We suppose that $\hat{F}^{L}(p), \hat{F}^{D}(p)$ and $\hat{F}^{W}(p)$ are bounded, non-negative and $\mathbb{G}$-adapted processes. 
Including partial withdrawals in the pay-off, we shall use the following notations

$$
\begin{aligned}
F_{\tau}^{D, W}(p):= & e^{-\int_{0}^{\tau} r_{u} d u}\left(\hat{F}_{\tau}^{D}(p) \mathbb{1}_{\left\{\tau=\theta^{d} \leq T\right\}}+\hat{F}_{\tau}^{W}(p) \mathbb{1}_{\left\{\tau=\theta^{w}<\theta^{d} ; \tau \leq T\right\}}\right) \\
& +\int_{0}^{\tau} \xi_{s} A_{s}^{p} d s, \\
F_{T}^{L}(p):= & e^{-\int_{0}^{T} r_{u} d u} \hat{F}_{T}^{L}(p)+\int_{0}^{T} \xi_{s} A_{s}^{p} d s \\
F(p):= & e^{-\int_{0}^{T \wedge \tau} r_{u} d u} \hat{F}(p)+\int_{0}^{T \wedge \tau} \xi_{s} A_{s}^{p} d s .
\end{aligned}
$$

Notice that $F(p)$ is $\mathcal{G}_{T \wedge \tau}$-measurable.

Usual examples of variable annuities are GMDB and GMLB. In that case, there exist $\hat{G}^{D}(p)$ and $\hat{G}^{L}(p)$ non-negative processes such that, for any $Q \in\{D, L\}$, we have

$$
\hat{F}_{t}^{Q}(p)=\hat{A}_{t}^{p} \vee \hat{G}_{t}^{Q}(p), \quad \text { where } \hat{A}_{t}^{p}=e^{\int_{0}^{t} r_{s} d s} A_{t}^{p} .
$$

The usual guarantee functions used to define GMDB and GMLB are listed below (see [2] for more details).

- Constant guarantee: we have $\hat{G}_{t}^{Q}(p)=A_{0}-\int_{0}^{t} \xi_{s} \hat{A}_{s}^{p} d s$ on $[0, T]$, and

$$
\begin{aligned}
F(p) & =A_{T \wedge \tau}^{p} \vee e^{-\int_{0}^{T \wedge \tau} r_{s} d s}\left(A_{0}-\int_{0}^{T \wedge \tau} \xi_{s} \hat{A}_{s}^{p} d s\right)+\int_{0}^{T \wedge \tau} \xi_{s} A_{s}^{p} d s \\
& =A_{T \wedge \tau}^{p}(0) \vee\left(e^{-\int_{0}^{T \wedge \tau} r_{s} d s} A_{0}+\int_{0}^{T \wedge \tau} \xi_{s} A_{s}^{p} \beta_{s} d s\right),
\end{aligned}
$$

where $A_{T \wedge \tau}^{p}(0)=A_{T \wedge \tau}^{p}+\int_{0}^{T \wedge \tau} \xi_{s} A_{s}^{p} d s$ and $\beta_{t}=1-e^{-\int_{t}^{T \wedge \tau} r_{s} d s}$ for $t \in[0, T \wedge \tau]$.

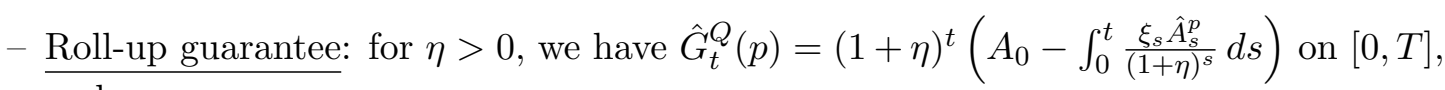
and

$$
\begin{aligned}
F(p) & =A_{T \wedge \tau}^{p} \vee e^{-\int_{0}^{T \wedge \tau} r_{s} d s}(1+\eta)^{T \wedge \tau}\left(A_{0}-\int_{0}^{T \wedge \tau} \frac{\xi_{s} \hat{A}_{s}^{p}}{(1+\eta)^{s}} d s\right)+\int_{0}^{T \wedge \tau} \xi_{s} A_{s}^{p} d s \\
& =A_{T \wedge \tau}^{p}(0) \vee\left(e^{-\int_{0}^{T \wedge \tau} r_{s}^{\eta} d s} A_{0}+\int_{0}^{T \wedge \tau} \xi_{s} A_{s}^{p} \beta_{s}^{\eta} d s\right),
\end{aligned}
$$

where $r_{t}^{\eta}=r_{t}-\ln (1+\eta)$ for all $t \in[0, T]$ and $\beta_{t}^{\eta}=1-e^{-\int_{t}^{T \wedge \tau} r_{s}^{\eta} d s}$ for $t \in[0, T \wedge \tau]$.

- Ratchet guarantee: the guarantee depends on the path of $A$ in the following way:

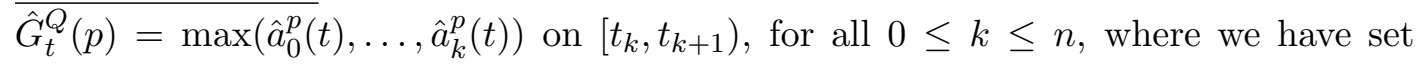




$$
\begin{aligned}
& \hat{a}_{k}^{p}(t)=\hat{A}_{t_{k}}^{p}-\int_{t_{k}}^{t} \xi_{s} \hat{A}_{s}^{p} d s . \text { We get } \\
& \begin{aligned}
F(p) & =A_{T \wedge \tau}^{p} \vee e^{-\int_{0}^{T \wedge \tau} r_{s} d s} \max _{0 \leq i \leq n}\left(\hat{a}_{i}^{p}(T \wedge \tau) \mathbb{1}_{\left\{t_{i} \leq T \wedge \tau\right\}}\right)+\int_{0}^{T \wedge \tau} \xi_{s} A_{s}^{p} d s \\
& =A_{T \wedge \tau}^{p}(0) \vee\left(\max _{0 \leq i \leq n}\left[e^{-\int_{0}^{T \wedge \tau} r_{s} d s} \hat{A}_{t_{i}}^{p}(0) \mathbb{1}_{\left\{t_{i} \leq T \wedge \tau\right\}}\right]+\int_{0}^{T \wedge \tau} \xi_{s} A_{s}^{p} \beta_{s} d s\right)(1 .
\end{aligned}
\end{aligned}
$$

where $\hat{A}_{t_{i}}^{p}(0)=\hat{A}_{t_{i}}^{p}+\int_{0}^{t_{i}} \xi_{s} \hat{A}_{s}^{p} d s$ for all $i \in\{0, . ., n\}$.

Remark 1.1. In the usual cases, the terminal pay-off $F(p)$ is non-increasing w.r.t. $p$.

At this point we also notice that, in usual cases, the pay-off $F(p)$ may not be bounded. This assumption is crucial from a mathematical point of view, since it leads to existence and uniqueness of a solution of the BSDEs that we will consider (see Remark 2.2). However, our methodology can be applied to such unbounded pay-offs. Indeed, from a numerical point of view, one just has to introduce a positive constant $m$ and replace the pay-off $F(p)$ by $F(p) \wedge m$. For $m$ large enough, we will get a good approximation of the indifference fee rate as $\lim _{m \rightarrow+\infty} \mathbb{P}\left(\sup _{t \in[0, T]} A_{t}>m\right)=0$. 
Figure 1 represents the evolution of roll-up and ratchet guarantees on a path.

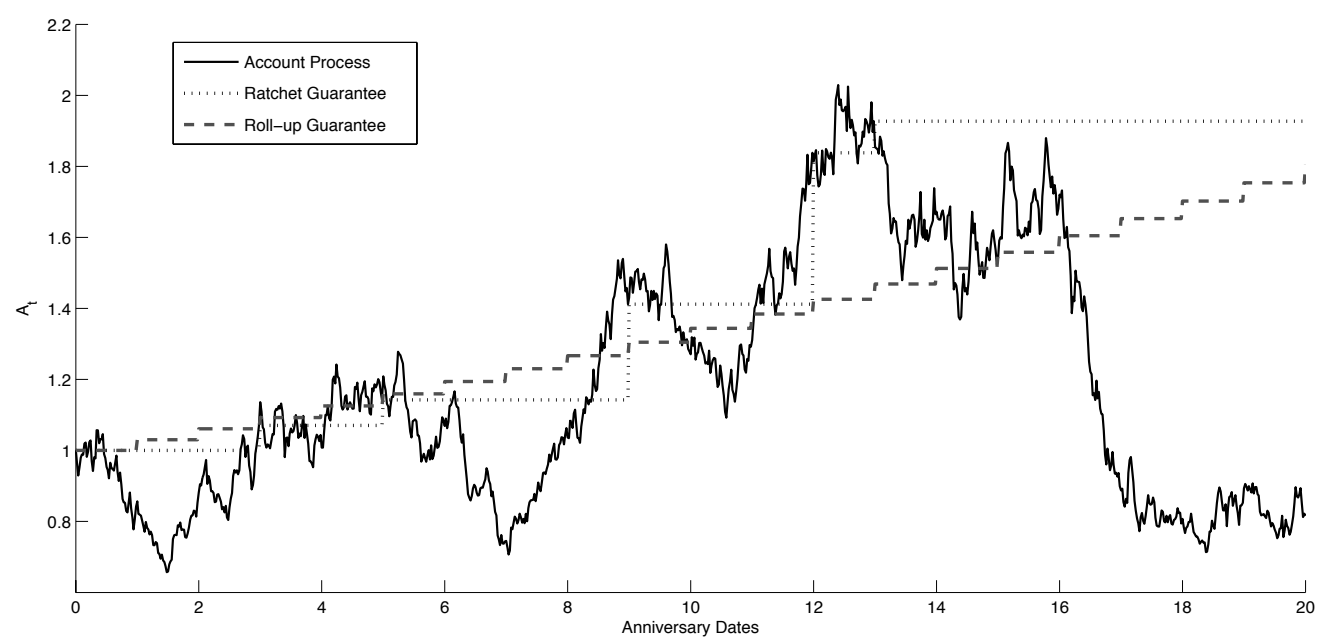

Figure 1: Guarantees and Account Value.

\section{Indifference pricing}

The objective of this section is to find, if it exists, a level $p^{*}$ such that if the fee rate is greater than $p^{*}$, the insurer prefers to sell the policy and he has better not to do so if the fee rate is below this level. To determine $p^{*}$ we have to study the solution of the following equation

$$
\sup _{\pi \in \mathcal{A}^{\mathbb{F}}[0, T]} \mathbb{E}\left[U\left(X_{T}^{x, \pi}\right)\right]=\sup _{\pi \in \mathcal{A}^{\mathbb{G}}[0, T]} \mathbb{E}\left[U\left(X_{T}^{x+A_{0}, \pi}-F\left(p^{*}\right)\right)\right]
$$

A solution of the equation (2.8) will be called an indifference fee rate. Notice that if there exist solutions to the previous equation, they will not depend on the initial wealth invested by the insurer but only on the initial deposit $A_{0}$ made by the insured since $U(y)=$ $-\exp (-\gamma y)$. Therefore, solve the equation $(2.8)$ is equivalent to solve

$$
\sup _{\pi \in \mathcal{A}^{\mathbb{F}}[0, T]} \mathbb{E}\left[U\left(X_{T}^{\pi}\right)\right]=\sup _{\pi \in \mathcal{A}^{\mathbb{G}}[0, T]} \mathbb{E}\left[U\left(A_{0}+X_{T}^{\pi}-F\left(p^{*}\right)\right)\right] .
$$

To solve this equation, we shall compute the following quantities

$$
V_{\mathbb{F}}:=\sup _{\pi \in \mathcal{A}^{\mathbb{F}}[0, T]} \mathbb{E}\left[U\left(X_{T}^{\pi}\right)\right] \quad \text { and } \quad V_{\mathbb{G}}(p):=\sup _{\pi \in \mathcal{A}^{\mathbb{G}}[0, T]} \mathbb{E}\left[U\left(A_{0}+X_{T}^{\pi}-F(p)\right)\right] .
$$

$V_{\mathbb{F}}$ is a classical optimization problem, that may be solved thanks to BSDEs like in [15] or [20]. We recall the results on this problem in Subsection 2.1, then in Subsection 2.2 we solve the optimal control problem $V_{\mathbb{G}}(p)$. We will use the tools of BSDEs with respect to 
the Brownian motion $B$ and to the jump process $N$ to solve it. Finally, in Subsection 2.3, we will use the results of Subsections 2.1 and 2.2 to find indifference fee rates if they exist. An additional difficulty with respect to the classical indifference pricing theory is that fees are continuously paid by the insured. Therefore, one can not use algebraic properties of the utility function to get a semi-explicit formula for the indifference fee rate. We will prove that the function $p \rightarrow V_{\mathbb{G}}(p)$ is continuous and monotonic on $\mathbb{R}$, then use the intermediate value theorem to prove that there exists or not a solution of the equation 2.8 .

\subsection{Utility maximization without variable annuities}

The objective of this part is to compute the value of the maximum expected utility of the terminal wealth at time $T$ when the insurance company has not sold the variable annuity policy. We recall that the maximum expected utility problem is defined by

$$
V_{\mathbb{F}}:=\sup _{\pi \in \mathcal{A}^{\mathbb{F}}[0, T]} \mathbb{E}\left[U\left(X_{T}^{\pi}\right)\right]
$$

Thanks to Theorem 7 in $\mathrm{Hu}$ et al. [15], we are able to characterize the value function $V_{\mathbb{F}}$ and the optimal strategy $\pi^{*}$ by mean of BSDEs. For that we introduce the following sets.

- $S_{\mathbb{G}}^{\infty}$ is the subset of $\mathbb{R}$-valued, càd-làg, $\mathbb{G}$-adapted processes $\left(Y_{t}\right)_{t \in[0, T]}$ essentially bounded i.e.

$$
\|Y\|_{S_{\mathbb{G}}^{\infty}}:=\left\|\sup _{t \in[0, T]}\left|Y_{t}\right|\right\|_{\infty}<\infty
$$

- $L_{\mathbb{G}}^{2}$ is the subset of $\mathbb{R}$-valued, $\mathbb{G}$-predictable processes $\left(Z_{t}\right)_{t \in[0, T]}$ such that

$$
\|Z\|_{L_{\mathbb{G}}^{2}}:=\left(\mathbb{E}\left[\int_{0}^{T}\left|Z_{t}\right|^{2} d t\right]\right)^{1 / 2}<\infty
$$

- $L_{\mathbb{G}}^{2}(\lambda)$ is the subset of $\mathbb{R}$-valued, $\mathbb{G}$-predictable processes $\left(U_{t}\right)_{t \in[0, T]}$ such that

$$
\|U\|_{L_{\mathbb{G}}^{2}(\lambda)}:=\left(\mathbb{E}\left[\int_{0}^{T \wedge \tau} \lambda_{t}\left|U_{t}\right|^{2} d t\right]\right)^{1 / 2}<\infty
$$

Proposition 2.1. The value function $V_{\mathbb{F}}$ is given by $V_{\mathbb{F}}=-\exp \left(\gamma y_{0}\right)$, where $(y, z)$ is the unique solution in $S_{\mathbb{G}}^{\infty} \times L_{\mathbb{G}}^{2}$ of the following BSDE

$$
\left\{\begin{array}{l}
d y_{t}=\left(\frac{\nu_{t}^{2}}{2 \gamma}+\nu_{t} z_{t}\right) d t+z_{t} d B_{t}, \quad \forall t \in[0, T] \\
y_{T}=0
\end{array}\right.
$$

with $\nu_{t}=\frac{\mu_{t}-r_{t}}{\sigma_{t}}$. Moreover, the optimal strategy associated to this problem is defined by

$$
\pi_{t}^{*}:=\frac{\nu_{t}}{\gamma \sigma_{t}}+\frac{z_{t}}{\sigma_{t}}, \quad \forall t \in[0, T]
$$

For the proof of this proposition we refer to [15] or [20]. 


\subsection{Utility maximization with variable annuities}

We now study the case in which the insurance company proposes the variable annuity policy. We recall that in this case the value function associated to the maximum expected utility problem is given by

$$
V_{\mathbb{G}}(p):=\sup _{\pi \in \mathcal{A}^{\mathbb{G}}[0, T]} \mathbb{E}\left[-\exp \left(-\gamma\left(A_{0}+X_{T}^{\pi}-F(p)\right)\right)\right],
$$

where $F(p)$ is defined by 1.4 .

Since we aim at characterizing $V_{\mathbb{G}}(p)$ as a function of the initial value of a BSDE, the first step consists in carefully setting the terminal value of the BSDE. Therefore, we need to deal with the following difficulty: we notice that the random variable $X_{T}^{\pi}$ is $\mathcal{G}_{T}$-measurable and $F(p)$ is $\mathcal{G}_{T \wedge \tau}$-measurable. The following result allows us to rewrite the problem with a terminal date equal to $T \wedge \tau$.

Lemma 2.1. For any $p \in \mathbb{R}$, we have

$$
V_{\mathbb{G}}(p)=\sup _{\pi \in \mathcal{A}^{\mathbb{G}}[0, T \wedge \tau]} \mathbb{E}\left[-\exp \left(-\gamma\left(X_{T \wedge \tau}^{A_{0}, \pi}-H(p)\right)\right)\right]
$$

with $H(p):=F(p)+\frac{1}{\gamma} \log \left\{\operatorname{ess}_{\inf _{\pi \in \mathcal{A}^{\mathbb{G}}[T \wedge \tau, T]}} \mathbb{E}\left[\exp \left(-\gamma \Delta X_{\tau, T}^{\pi}\right) \mid \mathcal{G}_{T \wedge \tau}\right]\right\}$, where we have set

$$
\Delta X_{\tau, T}^{\pi}:=\int_{T \wedge \tau}^{T} \pi_{s}\left(\mu_{s}-r_{s}\right) d s+\int_{T \wedge \tau}^{T} \pi_{s} \sigma_{s} d B_{s}
$$

Proof. First we prove that

$$
V_{\mathbb{G}}(p) \leq \sup _{\pi \in \mathcal{A}^{\mathbb{G}}[0, T \wedge \tau]} \mathbb{E}\left[-\exp \left(-\gamma\left(X_{T}^{A_{0}, \pi}-H(p)\right)\right)\right]
$$

Let $\pi^{\prime} \in \mathcal{A}^{\mathbb{G}}[0, T]$. By the tower property and since $F(p)$ is $\mathcal{G}_{T \wedge \tau}$-measurable, we get

$$
\begin{aligned}
\mathbb{E}\left[\exp \left(-\gamma\left(X_{T}^{A_{0}, \pi^{\prime}}-F(p)\right)\right)\right] & =\mathbb{E}\left[\exp \left(-\gamma\left(X_{T \wedge \tau}^{A_{0}, \pi^{\prime}}-F(p)\right)\right) \mathbb{E}\left[\exp \left(-\gamma \Delta X_{\tau, T}^{\pi^{\prime}}\right) \mid \mathcal{G}_{T \wedge \tau}\right]\right] \\
& \left.\geq \mathbb{E}\left[\exp \left(-\gamma\left(X_{T \wedge \tau}^{A_{0}, \pi^{\prime}}-F(p)\right)\right) \underline{V} \mid \mathcal{G}_{T \wedge \tau}\right]\right]
\end{aligned}
$$

where we have set

$$
\underline{V}:=\underset{\pi \in \mathcal{A}^{\mathbb{G}}[T \wedge \tau, T]}{\operatorname{essinf}} \mathbb{E}\left[\exp \left(-\gamma \Delta X_{\tau, T}^{\pi}\right) \mid \mathcal{G}_{T \wedge \tau}\right]
$$

Therefore, it follows from the definition of $H(p)$ that for any $\pi^{\prime} \in \mathcal{A}^{\mathbb{G}}[0, T]$, we have

$$
\mathbb{E}\left[\exp \left(-\gamma\left(X_{T}^{A_{0}, \pi^{\prime}}-F(p)\right)\right)\right] \geq \inf _{\pi \in \mathcal{A}^{\mathbb{G}}[0, T \wedge \tau]} \mathbb{E}\left[\exp \left(-\gamma\left(X_{T \wedge \tau}^{A_{0}, \pi}-H(p)\right)\right)\right] .
$$


This obviously implies that

$$
V_{\mathbb{G}}(p) \leq \sup _{\pi \in \mathcal{A}^{\mathbb{G}}[0, T \wedge \tau]} \mathbb{E}\left[-\exp \left(-\gamma\left(X_{T \wedge \tau}^{A_{0}, \pi}-H(p)\right)\right)\right]
$$

Now, we shall prove that

$$
V_{\mathbb{G}}(p) \geq \sup _{\pi \in \mathcal{A}^{\mathbb{G}}[0, T \wedge \tau]} \mathbb{E}\left[-\exp \left(-\gamma\left(X_{T}^{A_{0}, \pi}-H(p)\right)\right)\right]
$$

From Lemma A.1, we deduce that there exists $\pi^{*, \tau} \in \mathcal{A}^{\mathbb{G}}[T \wedge \tau, T]$ such that

$$
\mathbb{E}\left[\exp \left(-\gamma \Delta X_{\tau, T}^{\pi^{*, \tau}}\right) \mid \mathcal{G}_{T \wedge \tau}\right]=\operatorname{ess~inf}_{\pi \in \mathcal{A}^{\mathbb{G}}[T \wedge \tau, T]} \mathbb{E}\left[\exp \left(-\gamma \Delta X_{\tau, T}^{\pi}\right) \mid \mathcal{G}_{T \wedge \tau}\right] .
$$

For any $\pi \in \mathcal{A}^{\mathbb{G}}[0, T \wedge \tau]$ we define the strategy $\underline{\pi} \in \mathcal{A}^{\mathbb{G}}[0, T]$ by

$$
\underline{\pi}_{t}:= \begin{cases}\pi_{t} & \text { if } t \leq T \wedge \tau, \\ \pi_{t}^{*, \tau} & \text { if } t>T \wedge \tau .\end{cases}
$$

We obtain

$$
\begin{aligned}
V_{\mathbb{G}}(p) & \geq \sup _{\pi \in \mathcal{A}^{\mathbb{G}}[0, T \wedge \tau]} \mathbb{E}\left[-\exp \left(-\gamma\left(X_{T}^{A_{0}, \underline{\pi}}-F(p)\right)\right)\right] \\
& =\sup _{\pi \in \mathcal{A}^{\mathbb{G}}[0, T \wedge \tau]} \mathbb{E}\left[-\exp \left(-\gamma\left(X_{T \wedge \tau}^{A_{0}, \pi}+\Delta X_{\tau, T}^{\pi^{*, \tau}}-F(p)\right)\right)\right] \\
& =\sup _{\pi \in \mathcal{A}^{\mathbb{G}}[0, T \wedge \tau]} \mathbb{E}\left[-\exp \left(-\gamma\left(X_{T \wedge \tau}^{A_{0}, \pi}-H(p)\right)\right)\right] .
\end{aligned}
$$

Now, we have to solve the optimization problem (2.11) and for that we look for a family of processes $\left\{R^{(\pi)}, \pi \in \mathcal{A}^{\mathbb{G}}[0, T \wedge \tau]\right\}$ satisfying the following conditions

(i) $R_{T \wedge \tau}^{(\pi)}=-\exp \left(-\gamma\left(X_{T \wedge \tau}^{A_{0}, \pi}-H(p)\right)\right)$, for any $\pi \in \mathcal{A}^{\mathbb{G}}[0, T \wedge \tau]$.

(ii) $R_{0}^{(\pi)}=R_{0}$ is constant for any $\pi \in \mathcal{A}^{\mathbb{G}}[0, T \wedge \tau]$.

(iii) $R^{(\pi)}$ is a $\mathbb{G}$-supermartingale for any $\pi \in \mathcal{A}^{\mathbb{G}}[0, T \wedge \tau]$.

(iv) There exists a $\pi^{*} \in \mathcal{A}^{\mathbb{G}}[0, T \wedge \tau]$ such that $R^{\left(\pi^{*}\right)}$ is a $\mathbb{G}$-martingale.

If such a family exist, we would have

$$
R_{0}^{\left(\pi^{*}\right)}=\sup _{\pi \in \mathcal{A}^{\mathbb{G}}[0, T \wedge \tau]} \mathbb{E}\left[-\exp \left(-\gamma\left(X_{T \wedge \tau}^{A_{0}, \pi}-H(p)\right)\right)\right]
$$

Indeed, from (i), (ii) and (iii), we might have for any $\pi \in \mathcal{A}^{\mathbb{G}}[0, T \wedge \tau]$,

$$
R_{0}^{\left(\pi^{*}\right)}=R_{0}^{(\pi)} \geq \mathbb{E}\left[R_{T \wedge \tau}^{(\pi)}\right]=\mathbb{E}\left[-\exp \left(-\gamma\left(X_{T \wedge \tau}^{A_{0}, \pi}-H(p)\right)\right)\right] .
$$


Moreover, it would follow from (i) and (iv) that

$$
R_{0}^{\left(\pi^{*}\right)}=\mathbb{E}\left[-\exp \left(-\gamma\left(X_{T \wedge \tau}^{A_{0}, \pi^{*}}-H(p)\right)\right)\right] .
$$

Therefore, from 2.12 and 2.13 , we would get for any $\pi \in \mathcal{A}^{\mathbb{G}}[0, T \wedge \tau]$

$$
\mathbb{E}\left[-\exp \left(-\gamma\left(X_{T \wedge \tau}^{A_{0}, \pi}-H(p)\right)\right)\right] \leq R_{0}^{\left(\pi^{*}\right)}=\mathbb{E}\left[-\exp \left(-\gamma\left(X_{T \wedge \tau}^{A_{0}, \pi^{*}}-H(p)\right)\right)\right] .
$$

We can see that it would lead to

$$
R_{0}^{\left(\pi^{*}\right)}=\sup _{\pi \in \mathcal{A}^{\mathbb{G}}[0, T \wedge \tau]} \mathbb{E}\left[-\exp \left(-\gamma\left(X_{T \wedge \tau}^{A_{0}, \pi}-H(p)\right)\right)\right]
$$

Thanks to solutions of BSDEs with jumps, we shall construct a family $\left\{R^{(\pi)}, \pi \in \mathcal{A}^{\mathbb{G}}[0, T \wedge\right.$ $\tau]\}$ satisfying the previous conditions. Let $f$ be a function defined on $[0, T] \times \Omega \times S_{\mathbb{G}}^{\infty} \times$ $L_{\mathbb{G}}^{2} \times L_{\mathbb{G}}^{2}(\lambda)$ and assume that there exists $(Y(p), Z(p), U(p))$ in $S_{\mathbb{G}}^{\infty} \times L_{\mathbb{G}}^{2} \times L_{\mathbb{G}}^{2}(\lambda)$ solution of the following BSDE: for any $t \in[0, T]$,

$$
Y_{t}(p)=H(p)+\int_{t \wedge \tau}^{T \wedge \tau} f\left(s, Y_{s}(p), Z_{s}(p), U_{s}(p)\right) d s-\int_{t \wedge \tau}^{T \wedge \tau} Z_{s}(p) d B_{s}-\int_{t \wedge \tau}^{T \wedge \tau} U_{s}(p) d N_{s} .
$$

In this case, for any $\pi \in \mathcal{A}^{\mathbb{G}}[0, T \wedge \tau]$, we set

$$
R^{(\pi)}=-\exp \left(-\gamma\left(X^{A_{0}, \pi}-Y(p)\right)\right)
$$

and look for a function $f$ for which the family $\left\{R^{(\pi)}, \pi \in \mathcal{A}^{\mathbb{G}}[0, T \wedge \tau]\right\}$ satisfies the conditions (i), (ii), (iii) and (iv). In order to calculate $f$, we apply Itô's formula and get

$$
d R_{t}^{(\pi)}=d M_{t}^{\pi}+d K_{t}^{\pi}
$$

where $M^{\pi}$ and $K^{\pi}$ are defined by

$$
\begin{aligned}
d M_{t}^{\pi} & :=-\gamma R_{t}^{(\pi)}\left(\sigma_{t} \pi_{t}-Z_{t}(p)\right) d B_{t}+R_{t^{-}}^{(\pi)}\left(e^{\gamma U_{t}(p)}-1\right) d M_{t} \\
d K_{t}^{\pi} & :=-\gamma R_{t}^{(\pi)}\left[\pi_{t}\left(\mu_{t}-r_{t}\right)+f\left(t, Y_{t}(p), Z_{t}(p), U_{t}(p)\right)-\frac{\gamma}{2}\left(\sigma_{t} \pi_{t}-Z_{t}(p)\right)^{2}-\lambda_{t} \frac{e^{\gamma U_{t}(p)}-1}{\gamma}\right] d t .
\end{aligned}
$$

As we hope that $R^{(\pi)}$ is a supermartingale the process $K^{\pi}$ must be non-increasing, hence $f$ should satisfy

$$
-\gamma R_{t}^{(\pi)}\left[\pi_{t}\left(\mu_{t}-r_{t}\right)+f\left(t, Y_{t}(p), Z_{t}(p), U_{t}(p)\right)-\frac{\gamma}{2}\left(\sigma_{t} \pi_{t}-Z_{t}(p)\right)^{2}-\lambda_{t} \frac{e^{\gamma U_{t}(p)}-1}{\gamma}\right] \leq 0
$$

and since $-\gamma R_{t}^{(\pi)} \geq 0$, it would lead to

$$
f\left(t, Y_{t}(p), Z_{t}(p), U_{t}(p)\right) \leq \frac{\gamma}{2}\left(\sigma_{t} \pi_{t}-Z_{t}(p)\right)^{2}+\lambda_{t} \frac{e^{\gamma U_{t}(p)}-1}{\gamma}-\pi_{t}\left(\mu_{t}-r_{t}\right)
$$


Moreover, for some particular $\pi^{*} \in \mathcal{A}^{\mathbb{G}}[0, T \wedge \tau]$, we hope that $R^{\left(\pi^{*}\right)}$ is a martingale so the process $K^{\pi^{*}}$ must be constant, hence $f$ should satisfy

$f\left(t, Y_{t}(p), Z_{t}(p), U_{t}(p)\right)=\operatorname{ess}_{\pi \in \mathcal{A}^{\mathbb{G}}[0, T \wedge \tau]}\left\{\frac{\gamma}{2}\left(\sigma_{t} \pi_{t}-Z_{t}(p)\right)^{2}+\lambda_{t} \frac{e^{\gamma U_{t}(p)}-1}{\gamma}-\pi_{t}\left(\mu_{t}-r_{t}\right)\right\}$,

and $\pi_{t}^{*}$, such that $d K_{t}^{\pi^{*}}=0$, would be defined by

$$
\pi_{t}^{*}:=\frac{\nu_{t}}{\gamma \sigma_{t}}+\frac{Z_{t}(p)}{\sigma_{t}}
$$

Hence $f$ would be the following function

$$
f(t, y, z, u)=\lambda_{t} \frac{e^{\gamma u}-1}{\gamma}-\frac{\nu_{t}^{2}}{2 \gamma}-\nu_{t} z
$$

defined on $[0, T] \times \Omega \times S_{\mathbb{G}}^{\infty} \times L_{\mathbb{G}}^{2} \times L_{\mathbb{G}}^{2}(\lambda)$.

The following proposition asserts that the following BSDE with jump

$$
\begin{aligned}
Y_{t}(p)= & H(p)+\int_{t \wedge \tau}^{T \wedge \tau}\left(\lambda_{s} \frac{e^{\gamma U_{s}(p)}-1}{\gamma}-\frac{\nu_{s}^{2}}{2 \gamma}-\nu_{s} Z_{s}(p)\right) d s-\int_{t \wedge \tau}^{T \wedge \tau} Z_{s}(p) d B_{s} \\
& -\int_{t \wedge \tau}^{T \wedge \tau} U_{s}(p) d N_{s}, \quad \forall t \in[0, T]
\end{aligned}
$$

admits a solution in $S_{\mathbb{G}}^{\infty} \times L_{\mathbb{G}}^{2} \times L_{\mathbb{G}}^{2}(\lambda)$.

Proposition 2.2. Recalling notations (1.2), (1.3) and (1.4), the BSDE (2.15) admits a solution $(Y(p), Z(p), U(p)) \in S_{\mathbb{G}}^{\infty} \times L_{\mathbb{G}}^{2} \times L_{\mathbb{G}}^{2}(\lambda)$ given for any $t \in[0, T]$ by

$$
\left\{\begin{array}{l}
Y_{t}(p)=Y_{t}^{0}(p) \mathbb{1}_{t<\tau}+F_{\tau}^{D, W}(p) \mathbb{1}_{\tau \leq t} \\
Z_{t}(p)=Z_{t}^{0}(p) \mathbb{1}_{t \leq \tau} \\
U_{t}(p)=\left(F_{t}^{D, W}(p)-Y_{t}^{0}(p)\right) \mathbb{1}_{t \leq \tau}
\end{array}\right.
$$

where $\left(Y^{0}(p), Z^{0}(p)\right)$ is the unique solution in $S_{\mathbb{G}}^{\infty} \times L_{\mathbb{G}}^{2}$ of the following BSDE

$$
\left\{\begin{aligned}
-d Y_{t}^{0}(p) & =\left\{\lambda_{t} \frac{e^{\gamma\left(F_{t}^{D, W}(p)-Y_{t}^{0}(p)\right)}-1}{\gamma}-\frac{\nu_{t}^{2}}{2 \gamma}-\nu_{t} Z_{t}^{0}(p)\right\} d t-Z_{t}^{0}(p) d B_{t}, \\
Y_{T}^{0}(p) & =F_{T}^{L}(p) .
\end{aligned}\right.
$$

Proof. From Theorem 2.1 in [6] and Theorem 1 in [14], we know that there is a unique solution $\left(Y^{0}(p), Z^{0}(p)\right) \in S_{\mathbb{G}}^{\infty} \times L_{\mathbb{G}}^{2}$ to the BSDE (2.17).

From Theorem 4.3 in [17, we know that $(Y(p), Z(p), U(p))$ defined by 2.16 is a solution of the BSDE 2.15).

Remark 2.2. To apply Theorem 2.1 in [6] or Theorem 1 in [14] and get existence result for a solution of the BSDE (2.17), the terminal condition $F_{T}^{L}(p)$ must be bounded and the process $F^{D, W}(p)$ must be also bounded. 
We conclude this section with its main result which is the following verification theorem.

Theorem 2.1. The value function of the optimization problem (2.10) is given by

$$
V_{\mathbb{G}}(p)=-\exp \left(\gamma\left(Y_{0}(p)-A_{0}\right)\right),
$$

where $Y_{0}(p)$ is defined by the initial value of the first component of the solution of the BSDE 2.15 defined in Proposition 2.2.

Moreover there exists an optimal strategy $\pi^{*} \in \mathcal{A}^{\mathbb{G}}[0, T]$ and this one is defined by

$$
\pi_{t}^{*}:=\frac{\nu_{t}}{\gamma \sigma_{t}}+\frac{Z_{t}(p)}{\sigma_{t}} \mathbb{1}_{t \leq T \wedge \tau}+\frac{Z_{t}^{(\tau)}}{\sigma_{t}} \mathbb{1}_{t>T \wedge \tau}, \quad \forall t \in[0, T],
$$

with $Z(p)$ (resp. $\left.Z^{(\tau)}\right)$ defined by the solution of the BSDE 2.15 described in Proposition 2.2 (resp. Lemma A.1).

Notice that $Y_{0}(p)=Y_{0}^{0}(p)$ since the insurer can not withdraw his money at time 0 .

In the proof of Theorem 2.1, the additional space of BMO-martingales intervenes: $\mathrm{BMO}(\mathbb{P})$ is the subset of $(\mathbb{P}, \mathbb{G})$-martingales $m$ such that

$$
\|m\|_{\mathrm{BMO}(\mathbb{P})}:=\sup _{\theta \in \mathcal{T}_{\mathbb{G}}[0, T]}\left\|\mathbb{E}\left[\langle m\rangle_{T}-\langle m\rangle_{\theta} \mid \mathcal{G}_{\theta}\right]^{1 / 2}\right\|_{\infty}<\infty
$$

where $\mathcal{T}_{\mathbb{G}}[0, T]$ is the set of $\mathbb{G}$-stopping times on $[0, T]$.

Before proving Theorem 2.1, we need the following lemma.

Lemma 2.2. Let $\left(Y^{0}(p), Z^{0}(p)\right) \in S_{\mathbb{G}}^{\infty} \times L_{\mathbb{G}}^{2}$ be the solution of the BSDE (2.17), and let $\pi^{*}$ be the strategy given by (2.18). The processes $\int_{0}^{*} Z_{s}^{0}(p) d B_{s}$ and $\int_{0}^{*} \sigma_{s} \pi_{s}^{*} d B_{s}$ are $\mathrm{BMO}(\mathbb{P})$ martingales.

The proof of this technical lemma is given in Appendix A.2.

Corollary 2.1. The strategy $\pi^{*}$ defined in 2.18 belongs to $\mathcal{A}^{\mathbb{G}}[0, T]$.

Proof. $\pi^{*}$ is $\mathbb{G}$-measurable by definition, now using Assumption A1 and 2.2 we have that

$$
\begin{aligned}
\mathbb{E}\left[\int_{0}^{T}\left|\pi_{s}^{*} \sigma_{s}\right|^{2} d s\right] & =\mathbb{E}\left[\int_{0}^{T \wedge \tau}\left(\frac{\nu_{t}}{\gamma}-Z_{t}(p)\right)^{2} d t\right]+\mathbb{E}\left[\int_{T \wedge \tau}^{T}\left(\frac{\nu_{t}}{\gamma}-Z_{t}^{(\tau)}\right)^{2} d t\right] \\
& \leq c+c \mathbb{E}\left[\int_{0}^{T \wedge \tau}\left|Z_{t}(p)\right|^{2} d t\right]+c \mathbb{E}\left[\int_{T \wedge \tau}^{T}\left|Z_{t}^{(\tau)}\right|^{2} d t\right] \\
& <\infty,
\end{aligned}
$$

where $c$ is a positive constant.

It follows from Lemma 2.2 and properties of BMO-martingales (see for example [16]) that the family

$$
\left\{-\exp \left(-\gamma X_{\theta}^{\pi^{*}}\right), \theta \text { is a } \mathbb{G} \text {-stopping time with values in }[0, T]\right\}
$$


is uniformly integrable.

Now, we are able to prove Theorem 2.1.

Proof. First we check that the family $\left\{R^{(\pi)}, \pi \in \mathcal{A}^{\mathbb{G}}[0, T]\right\}$ defined in 2.14 satisfies properties (ii), (ii), (iii) and (iv).

Properties (i) and (iii) directly follow from the definition of $R^{(\pi)}$. To prove that condition (iii) is satisfied, we apply Itô's formula and get

$$
\begin{aligned}
d R_{t}^{(\pi)}= & -\gamma R_{t}^{(\pi)}\left[\pi_{t}\left(\mu_{t}-r_{t}\right)-\frac{\nu_{t}^{2}}{2 \gamma}-\nu_{t} Z_{t}(p)-\frac{\gamma}{2}\left(\sigma_{t} \pi_{t}-Z_{t}(p)\right)^{2}\right] d t \\
& -\gamma R_{t}^{(\pi)}\left(\sigma_{t} \pi_{t}-Z_{t}(p)\right) d B_{t}+R_{t^{-}}^{(\pi)}\left(e^{\gamma U_{t}(p)}-1\right) d M_{t} .
\end{aligned}
$$

This last equation has an explicit solution given by

$$
\begin{aligned}
R_{t}^{(\pi)}= & R_{0} \mathcal{E}\left(\int_{0}^{t} \gamma\left(Z_{s}(p)-\pi_{s} \sigma_{s}\right) d B_{s}+\int_{0}^{t}\left(e^{\gamma U_{s}(p)}-1\right) d M_{s}\right) \\
& \times \exp \left(-\gamma \int_{0}^{t}\left(\pi_{s}\left(\mu_{s}-r_{s}\right)-\frac{\nu_{s}^{2}}{2 \gamma}-\nu_{s} Z_{s}(p)-\frac{\gamma}{2}\left(\sigma_{s} \pi_{s}-Z_{s}(p)\right)^{2}\right) d s\right),
\end{aligned}
$$

where $\mathcal{E}$ denotes the Dolean-Dade exponential. Since $\pi \in \mathcal{A}^{\mathbb{G}}[0, T]$, the process $M^{\pi}:=$ $\mathcal{E}\left(\int_{0}^{\cdot} \gamma\left(Z_{t}(p)-\sigma_{t} \pi_{t}\right) d B_{t}+\left(e^{\gamma U_{t}(p)}-1\right) d M_{t}\right)$ is a local martingale. Hence, there exists a sequence of $\mathbb{G}$-stopping times $\left(\theta_{n}\right)_{n \in \mathbb{N}}$ satisfying $\lim _{n \rightarrow \infty} \theta_{n}=T \wedge \tau \mathbb{P}-$ a.s. and such that $M_{. \wedge \theta_{n}}^{\pi}$ is a positive martingale for each $n \in \mathbb{N}$. Moreover, since

$$
f\left(t, Y_{t}(p), Z_{t}(p), U_{t}(p)\right) \leq \frac{\gamma}{2}\left(\sigma_{t} \pi_{t}-Z_{t}(p)\right)^{2}+\lambda_{t} \frac{e^{\gamma U_{t}(p)}-1}{\gamma}-\pi_{t}\left(\mu_{t}-r_{t}\right)
$$

the process $\exp \left(-\gamma \int_{0}\left(\pi_{s}\left(\mu_{s}-r_{s}\right)-\frac{\nu_{s}^{2}}{2 \gamma}-Z_{s}(p) \nu_{s}-\frac{\gamma}{2}\left(\sigma_{s} \pi_{s}-Z_{s}(p)\right)^{2}\right) d s\right)$ is non-decreasing. As $R_{0}<0$, we get that $R_{. \wedge \theta_{n}}^{(\pi)}$ is a supermartingale and, for any $0 \leq s \leq t \leq T$, we have

$$
\mathbb{E}\left[R_{t \wedge \theta_{n}}^{(\pi)} \mid \mathcal{G}_{s}\right] \leq R_{s \wedge \theta_{n}}^{(\pi)} .
$$

This implies that, for any set $A \in \mathcal{G}_{s}$, we have the following inequality

$$
\mathbb{E}\left[R_{t \wedge \theta_{n}}^{(\pi)} \mathbb{1}_{A}\right] \leq \mathbb{E}\left[R_{s \wedge \theta_{n}}^{(\pi)} \mathbb{1}_{A}\right]
$$

Since $\pi$ is admissible and $Y$ is bounded, we remark that $\left(R_{t \wedge \theta_{n}}^{(\pi)}\right)_{n \in \mathbb{N}}$ and $\left(R_{s \wedge \theta_{n}}^{(\pi)}\right)_{n \in \mathbb{N}}$ are uniformly integrable, hence we may let $n$ goes to $+\infty$ and get

$$
\mathbb{E}\left[R_{t}^{(\pi)} \mathbb{1}_{A}\right] \leq \mathbb{E}\left[R_{s}^{(\pi)} \mathbb{1}_{A}\right], \quad \forall A \in \mathcal{G}_{s}
$$

This implies the claimed supermartingale property of $R^{(\pi)}$.

Finally, we know from Corollary 2.1 that $\pi^{*}$ is admissible and from construction of $\pi^{*}$, we have $R^{\left(\pi^{*}\right)}=M^{\pi^{*}}$, therefore $R^{\left(\pi^{*}\right)}$ is a martingale. This proves that condition (iv) is satisfied. 
Hence, for any $\pi \in \mathcal{A}^{\mathbb{G}}[0, T \wedge \tau]$, we obtain that

$$
\mathbb{E}\left[-\exp \left(-\gamma\left(X_{T \wedge \tau}^{A_{0}, \pi}-H(p)\right)\right)\right] \leq R_{0}^{(\pi)}=R_{0}^{\left(\pi^{*}\right)}=\mathbb{E}\left[-\exp \left(-\gamma\left(X_{T \wedge \tau}^{A_{0}, \pi^{*}}-H(p)\right)\right)\right] .
$$

Therefore, $V_{\mathbb{G}}(p)=-\exp \left(\gamma\left(Y_{0}(p)-A_{0}\right)\right)$ and $\pi^{*}$ is an optimal admissible strategy.

\subsection{Indifference fee rate}

In this section, our goal is to determine indifference fee rates i.e. positive numbers $p^{*}$ such that

$$
\sup _{\pi \in \mathcal{A}^{\mathbb{G}}[0, T]} \mathbb{E}\left[-\exp \left(-\gamma\left(X_{T}^{A_{0}, \pi}-F\left(p^{*}\right)\right)\right)\right]=\sup _{\pi \in \mathcal{A}^{\mathbb{F}}[0, T]} \mathbb{E}\left[-\exp \left(-\gamma X_{T}^{\pi}\right)\right] .
$$

It follows from results of Subsections 2.1 and 2.2 that the previous equation can be rewritten in the following way

$$
Y_{0}\left(p^{*}\right)-A_{0}=y_{0}
$$

To study this equation we introduce the function $\psi: \mathbb{R} \rightarrow \mathbb{R}$ defined as follows

$$
\psi(p):=Y_{0}(p)-y_{0}-A_{0}, \quad \forall p \in \mathbb{R}
$$

There may exist three cases depending on the coefficients values.

(i) For any $p \in \mathbb{R}$, we have $\psi(p)>0$. That means that, for any fee rate $p$, we have

$$
V_{\mathbb{G}}(p)<V_{\mathbb{F}}
$$

Therefore, the insurer's expected utility is always lower if he sells the variable annuities. Thus, he should not sell it.

(ii) For any $p \in \mathbb{R}$, we have $\psi(p)<0$. That means that, for any fee rate $p$, we have

$$
V_{\mathbb{G}}(p)>V_{\mathbb{F}}
$$

Therefore, the insurer's expected utility is always higher if he sells the variable annuities. Thus, he should sell it whatever the fees are.

(iii) There exist $p_{1}$ and $p_{2}$ such that $\psi\left(p_{1}\right) \psi\left(p_{2}\right)<0$. In this case, we prove in the remainder of this section that there exist indifference fee rates thanks to the intermediate value theorem applied to the function $\psi$.

We now give useful analytical properties of the function $\psi$.

Proposition 2.3. The function $\psi$ is continuous and non-increasing on $\mathbb{R}$.

Proof. We first show that $\psi$ is non-increasing. Let $p_{1}, p_{2} \in \mathbb{R}$ with $p_{1} \leq p_{2}$. By definition of the process $A^{p}$, for any $t \in[0, T]$, we have

$$
A_{t}^{p_{1}} \geq A_{t}^{p_{2}} \mathbb{P}-\text { a.s. }
$$


It follows from the monotonicity of $\hat{F}^{L}, \hat{F}^{D}$ and $\hat{F}^{W}$ that $F\left(p_{1}\right) \geq F\left(p_{2}\right) \mathbb{P}$-a.s. Hence, for any $\pi \in \mathcal{A}^{\mathbb{G}}[0, T]$, we have

$$
\mathbb{E}\left[-\exp \left(-\gamma\left(X_{T}^{A_{0}, \pi}-F\left(p_{1}\right)\right)\right)\right] \leq \mathbb{E}\left[-\exp \left(-\gamma\left(X_{T}^{A_{0}, \pi}-F\left(p_{2}\right)\right)\right)\right] .
$$

Since this inequality holds for any $\pi \in \mathcal{A}^{\mathbb{G}}[0, T]$, we get

$$
V_{\mathbb{G}}\left(p_{1}\right) \leq V_{\mathbb{G}}\left(p_{2}\right)
$$

As $V_{\mathbb{G}}(p)=-\exp \left(\gamma\left(Y_{0}(p)-A_{0}\right)\right)$ and $\gamma>0$, it follows that $\psi$ is non-increasing.

We now prove that $\psi$ is continuous on $\mathbb{R}$. For that we prove that the solution of the BSDE 2.17) is continuous w.r.t. the terminal condition by adapting a usual approach presented for example in [13]. Let $p_{1}<p_{2}$ and consider the solutions $\left(Y^{0}\left(p_{1}\right), Z^{0}\left(p_{1}\right)\right)$ and $\left(Y^{0}\left(p_{2}\right), Z^{0}\left(p_{2}\right)\right)$ associated to the BSDE 2.17) with respectively parameters $p_{1}$ and $p_{2}$. We define the processes $\delta Y\left(p_{1}, p_{2}\right):=Y^{0}\left(p_{2}\right)-Y^{0}\left(p_{1}\right)$ and $\delta Z\left(p_{1}, p_{2}\right):=Z^{0}\left(p_{2}\right)-Z^{0}\left(p_{1}\right)$. By applying Itï $; \frac{1}{2}$ 's formula to the process $\left(e^{\alpha t}\left|\delta Y_{t}\left(p_{1}, p_{2}\right)\right|^{2}\right)_{0 \leq t \leq T}$, we get that, for any $\alpha>0$,

$$
\begin{aligned}
d\left(e^{\alpha t}\left|\delta Y_{t}\left(p_{1}, p_{2}\right)\right|^{2}\right)= & \alpha e^{\alpha t}\left|\delta Y_{t}\left(p_{1}, p_{2}\right)\right|^{2} d t+2 e^{\alpha t} \delta Y_{t}\left(p_{1}, p_{2}\right) d\left(\delta Y_{t}\left(p_{1}, p_{2}\right)\right) \\
& +e^{\alpha t}\left|\delta Z_{t}\left(p_{1}, p_{2}\right)\right|^{2} d t .
\end{aligned}
$$

By usual arguments, we get

$$
\begin{aligned}
& e^{\alpha t}\left|\delta Y_{t}\left(p_{1}, p_{2}\right)\right|^{2}+\mathbb{E}\left[\int_{t}^{T} e^{\alpha s}\left|\delta Z_{s}\left(p_{1}, p_{2}\right)\right|^{2} d s \mid \mathcal{F}_{t}\right] \leq \\
& \mathbb{E}\left[e^{\alpha T}\left|\delta Y_{T}\left(p_{1}, p_{2}\right)\right|^{2}-\alpha \int_{t}^{T} e^{\alpha s}\left|\delta Y_{s}\left(p_{1}, p_{2}\right)\right|^{2} d s-2 \int_{t}^{T} e^{\alpha s} \delta Y_{s}\left(p_{1}, p_{2}\right) \delta Z_{s}\left(p_{1}, p_{2}\right) d s \mid \mathcal{F}_{t}\right] \\
& +\frac{2}{\gamma} \mathbb{E}\left[\int_{t}^{T} \lambda_{s} e^{\alpha s} \delta Y_{s}\left(p_{1}, p_{2}\right)\left(e^{\gamma\left(F_{s}^{D, W}\left(p_{2}\right)-Y_{s}\left(p_{2}\right)\right.}-e^{\gamma\left(F_{s}^{D, W}\left(p_{1}\right)-Y_{s}\left(p_{1}\right)\right)}\right) \mid \mathcal{F}_{t}\right] .
\end{aligned}
$$

By using Young's inequality, we get

$$
\begin{aligned}
e^{\alpha t}\left|\delta Y_{t}\left(p_{1}, p_{2}\right)\right|^{2} & \leq \mathbb{E}\left[e^{\alpha T}\left|\delta Y_{T}\left(p_{1}, p_{2}\right)\right|^{2} \mid \mathcal{F}_{t}\right]+(1-\alpha) \mathbb{E}\left[\int_{t}^{T} e^{\alpha s}\left|\delta Y_{s}\left(p_{1}, p_{2}\right)\right|^{2} d s \mid \mathcal{F}_{t}\right] \\
+ & \frac{2}{\gamma} \mathbb{E}\left[\int_{t}^{T} \lambda_{s} e^{\alpha s} \delta Y_{s}\left(p_{1}, p_{2}\right)\left(e^{\gamma\left(F_{s}^{D, W}\left(p_{2}\right)-Y_{s}\left(p_{2}\right)\right.}-e^{\gamma\left(F_{s}^{D, W}\left(p_{1}\right)-Y_{s}\left(p_{1}\right)\right)}\right) \mid \mathcal{F}_{t}\right] .
\end{aligned}
$$

Moreover, we know that $Y\left(p_{1}\right)$ and $Y\left(p_{2}\right)$ are lower bounded, hence there exists a constant $k$ such that $Y_{t}\left(p_{1}\right) \geq k$ and $Y_{t}\left(p_{2}\right) \geq k$ for any $t \in[0, T]$. Since the function $\exp (-\gamma(y \vee k))$ is Lipschitz continuous, the process $\lambda$ is bounded and the processes $F^{D, W}\left(p_{1}\right)$ and $F^{D, W}\left(p_{2}\right)$ are bounded, we can assert that there exists a positive constant $C$ such that

$$
e^{\alpha t}\left|\delta Y_{t}\left(p_{1}, p_{2}\right)\right|^{2} \leq \mathbb{E}\left[e^{\alpha T}\left|\delta Y_{T}\left(p_{1}, p_{2}\right)\right|^{2} \mid \mathcal{F}_{t}\right]+(C-\alpha) \mathbb{E}\left[\int_{t}^{T} e^{\alpha s}\left|\delta Y_{s}\left(p_{1}, p_{2}\right)\right|^{2} d s \mid \mathcal{F}_{t}\right]
$$


Hence, for $\alpha=C$, we get

$$
\left|\delta Y_{0}\left(p_{1}, p_{2}\right)\right|^{2} \leq \mathbb{E}\left[e^{C T}\left|\delta Y_{T}\left(p_{1}, p_{2}\right)\right|^{2}\right]
$$

We conclude the proof by recalling that $Y_{T}^{0}(p)$, the terminal condition to the BSDE (2.17), is continuous on $\mathbb{R}$ w.r.t. $p$ as we have assumed that the function $\hat{F}^{L}$ is continuous on $\mathbb{R}$.

We now consider the cases of usual guarantees.

Corollary 2.2. Ratchet guarantee

Let $m>A_{0}$. Recalling notations of the equation (1.7), we assume that

$$
F(p)=m \wedge\left[A_{T \wedge \tau}^{p}(0) \vee\left(\max _{0 \leq i \leq n}\left[e^{-\int_{0}^{T \wedge \tau} r_{s} d s} \hat{A}_{t_{i}}^{p}(0) \mathbb{1}_{\left\{t_{i} \leq T \wedge \tau\right\}}\right]+\int_{0}^{T \wedge \tau} \xi_{s} A_{s}^{p} \beta_{s} d s\right)\right]
$$

There exists $p^{*} \in \mathbb{R} \cup\{-\infty\}$ such that for $p \geq p^{*}$ we have $V_{\mathbb{G}}(p) \geq V_{\mathbb{F}}$ and for $p<p^{*}$ we have $V_{\mathbb{G}}(p)<V_{\mathbb{F}}$.

Proof. From Proposition 2.3, we just have to show that $\lim _{p \rightarrow+\infty} \psi(p) \leq 0$.

It would follow from the intermediate value theorem and the monotonicity of $\psi$ that there exists $p^{*} \in \mathbb{R} \cup\{-\infty\}$ such that $\psi(p) \leq 0$ for $p \geq p^{*}$ and $\psi(p)>0$ for $p<p^{*}$. First, notice that we may deduce from Assumption A1 that there exists a positive constant $C$ such that, for any $t \in[0, T], \mathbb{E}\left[A_{t}^{p}\right] \leq C e^{-p t}$. Therefore, as $A_{t}^{p} \geq 0$, we get

$$
\lim _{p \rightarrow+\infty} A_{t}^{p}=0 \quad \text { a.s. for any } t \in(0, T] .
$$

We now study the limit of $\psi$ at $+\infty$. We have

$$
\psi(p)+y_{0}=\frac{1}{\gamma} \ln \left(-V_{\mathbb{G}}(p)\right) .
$$

On the other hand, for any $\pi \in \mathcal{A}^{\mathbb{G}}[0, T]$, we have

$$
V_{\mathbb{G}}(p) \geq \mathbb{E}\left[-\exp \left(-\gamma\left(X_{T}^{\pi}+A_{0}-F(p)\right)\right]\right.
$$

Hence, it follows from the monotone convergence theorem that

$$
\begin{aligned}
\lim _{p \rightarrow+\infty} \psi(p)+y_{0} & =\frac{1}{\gamma} \ln \left(-\lim _{p \rightarrow+\infty} V_{\mathbb{G}}(p)\right) \\
& \leq \frac{1}{\gamma} \ln \left(\mathbb{E}\left[\exp \left(-\gamma\left(X_{T}^{\pi}+A_{0}-\lim _{p \rightarrow+\infty} F(p)\right)\right]\right)\right. \\
& =\frac{1}{\gamma} \ln \left(\mathbb{E}\left[\exp \left(-\gamma\left(X_{T}^{\pi}+A_{0}\left(1-e^{-\int_{0}^{T \wedge \tau} r_{s} d s}\right)\right)\right]\right)\right. \\
& \leq \frac{1}{\gamma} \ln \left(\mathbb{E}\left[\exp \left(-\gamma X_{T}^{\pi}\right)\right]\right) .
\end{aligned}
$$

We recall that $y_{0}=\frac{1}{\gamma} \ln \left(-V_{\mathbb{F}}\right)$ and that, from Proposition 2.1, there exists $\pi^{*} \in \mathcal{A}^{\mathbb{F}}[0, T] \subset$ $\mathcal{A}^{\mathbb{G}}[0, T]$, such that $y_{0}=\frac{1}{\gamma} \ln \left(\mathbb{E}\left[\exp \left(-\gamma X_{T}^{\pi^{*}}\right)\right]\right)$. Therefore, we obtain that $\lim _{p \rightarrow+\infty} \psi(p) \leq$ 
0 if we choose $\pi^{*}$ in 2.19 .

Corollary 2.3. Roll-up guarantee

Let $m>A_{0}$. Recalling notations of the equation (1.6), we assume that

$$
F(p)=m \wedge\left[A_{T \wedge \tau}^{p}(0) \vee\left(e^{-\int_{0}^{T \wedge \tau} r_{s}^{\eta} d s} A_{0}+\int_{0}^{T \wedge \tau} \xi_{s} A_{s}^{p} \beta_{s}^{\eta} d s\right)\right] .
$$

There exists $\eta_{*} \geq 0$ such that for any $\eta \in\left[0, \eta_{*}\right]$, there exists $p^{*} \in \mathbb{R} \cup\{-\infty\}$ such that for $p \geq p^{*}$ we have $V_{\mathbb{G}}(p) \geq V_{\mathbb{F}}$ and for $p<p^{*}$ we have $V_{\mathbb{G}}(p)<V_{\mathbb{F}}$.

Proof. Let $\eta \geq 0$. From Proposition 2.1. there exists $\pi^{*} \in \mathcal{A}^{\mathbb{F}}[0, T] \subset \mathcal{A}^{\mathbb{G}}[0, T]$, such that $y_{0}=\frac{1}{\gamma} \ln \left(\mathbb{E}\left[\exp \left(-\gamma X_{T}^{\pi^{*}}\right)\right]\right)$. Following the proof of Corollary 2.2, we deduce from the monotone convergence theorem that

$$
\begin{aligned}
\lim _{p \rightarrow+\infty} \psi(p)+y_{0} & =\frac{1}{\gamma} \ln \left(-\lim _{p \rightarrow+\infty} V_{\mathbb{G}}(p)\right) \\
& \leq \frac{1}{\gamma} \ln \left(\mathbb{E}\left[\exp \left(-\gamma\left(X_{T}^{\pi^{*}}+A_{0}-\lim _{p \rightarrow+\infty} F(p)\right)\right)\right]\right) \\
& =\frac{1}{\gamma} \ln (\Phi(\eta)),
\end{aligned}
$$

where we have set

$$
\Phi(\eta):=\mathbb{E}\left[\exp \left(-\gamma\left(X_{T}^{\pi^{*}}+A_{0}\left(1-e^{-\int_{0}^{T \wedge \tau} r_{s}^{\eta} d s}\right)\right)\right)\right] .
$$

Obviously, $\Phi$ is continuous and non-decreasing on $\mathbb{R}^{+}$. Moreover, we have

$$
\Phi(0) \leq \mathbb{E}\left[\exp \left(-\gamma X_{T}^{\pi^{*}}\right)\right]=e^{\gamma y_{0}} \quad \text { and } \quad \lim _{\eta \rightarrow+\infty} \Phi(\eta)=+\infty .
$$

From the intermediate value theorem, we may define $\eta_{*} \geq 0$ as

$$
\eta_{*}:=\sup \left\{\eta \geq 0, \Phi(\eta)=e^{\gamma y_{0}}\right\}
$$

We conclude the proof by noticing that for $0 \leq \eta \leq \eta_{*}$, we have

$$
\lim _{p \rightarrow+\infty} \psi(p) \leq \frac{1}{\gamma} \ln (\Phi(\eta))-y_{0} \leq 0 .
$$

\section{Simulations}

In this section we present numerical illustrations of parameters sensibility for indifference fee rates. We compute solutions for both optimization problems: $V_{\mathbb{F}}$, the utility maximization problem without variable annuities, and $V_{\mathbb{G}}(p)$, the utility maximization problem with variable annuities. We simulate the BSDEs involved, using the discretization scheme 
studied in [7]. For the computation of the conditional expectations, we use non-parametric regression method with the Gaussian function as kernel. Following a dichotomy method, we find $p$ such that the equality $V_{\mathbb{F}}=V_{\mathbb{G}}(p)$ is satisfied.

We assume that $r$ and $\mu$ are Markov chains taking values in the states spaces $S^{r}=$ $\{0,0.01, \ldots, 0.25\}$ and $S^{\mu}=\{0,0.01,0.02, \ldots, 0.3\}$. Their respective transitional matrix are $Q^{r}=\left\{q_{i, j}^{r}\right\}_{1 \leq i, j \leq 26}$ and $Q^{\mu}=\left\{q_{i, j}^{\mu}\right\}_{1 \leq i, j \leq 31}$ are given by

$q_{i, j}^{r}=\left\{\begin{array}{cc}\frac{1}{2} \quad \text { if } & i=j, \\ \frac{1}{2} \quad \text { if } & i=1 \text { and } j=2, \\ \frac{1}{2} \quad \text { if } & i=27 \text { and } j=26, \\ \frac{1}{4} \quad \text { if } \quad i=j+1 \text { and } i \leq 26, \\ \frac{1}{4} \quad \text { if } \quad i=j-1 \text { and } i \geq 2, \\ 0 \quad \text { else, }\end{array} \quad\right.$ and $\quad q_{i, j}^{\mu}=\left\{\begin{array}{ccc}\frac{1}{2} & \text { if } & i=j, \\ \frac{1}{2} & \text { if } & i=1 \text { and } j=2, \\ \frac{1}{2} & \text { if } & i=32 \text { and } j=31 \\ \frac{1}{4} & \text { if } & i=j+1 \text { and } i \leq 31, \\ \frac{1}{4} & \text { if } & i=j-1 \text { and } i \geq 2, \\ 0 & \text { else, }\end{array}\right.$

Initial values $\mu_{0}$ and $r_{0}$ will be precised later. For simplicity, we assume that there are no early withdrawals i.e. we set $\left(\xi_{t}\right)_{t \geq 0} \equiv 0$, except for Figure 9. We shall give the following numerical values to parameters

$$
\gamma=1.3, \quad \lambda=0.05, \quad T=20, \quad A_{0}=1,
$$

and, for the financial market parameters

$$
r_{0}=0.02, \quad \mu_{0}=0.15, \quad \sigma=0.3
$$

We divide our numerical study in three parts. First, we consider a product with a ratchet guarantee and describe the dependence with respect to the market parameters: the initial interest rate (see Figure 2), the initial drift (see Figure 3) and the volatility (see Figure 4). In a second part, still with ratchet guarantee, we give illustrations of the dependence with respect to the longevity parameters: the contract maturity and the exit time intensity. In the last part, we consider the case with a roll-up guarantee and compute the sensibilities of indifference fees to variations of the initial value $A_{0}$, the roll-up rate $\eta$ and finally to variations of the withdrawal rate $\xi$.

\subsection{Market risk}

In this first part, we want to understand the impact of market risks on the indifference fee. For that we consider the case of ratchet guarantees.

Figure 2 plots the indifference fee rates when the initial interest rate $r_{0}$ ranged from 0.01 to 0.055 . 


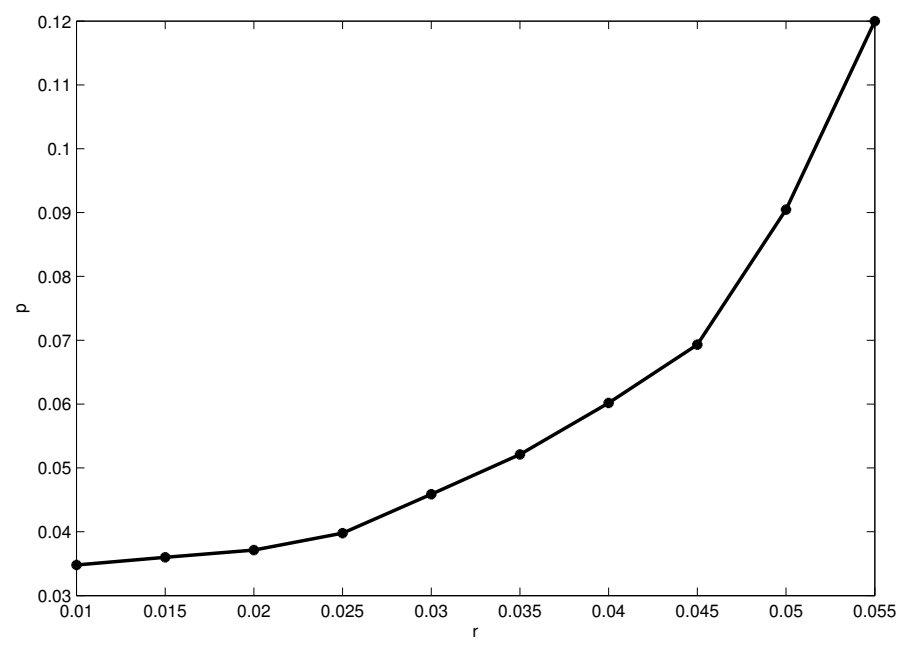

Figure 2: Indifference fee rate w.r.t. $r_{0}$

We notice that indifference fee rates increase with interest rate. This is due to the guarantee structure of the product: a growth of interest rate will lead to a growth of the quantity $V_{\mathbb{G}}(p)$ with respect to $V_{\mathbb{F}}$ and to compensate this growth we will have to increase $p$, as $p \rightarrow V_{\mathbb{G}}(p)$ is non-increasing.

Figure 3 plots the indifference fee rates when the initial drift $\mu_{0}$ ranged from 0.02 to 0.3 .

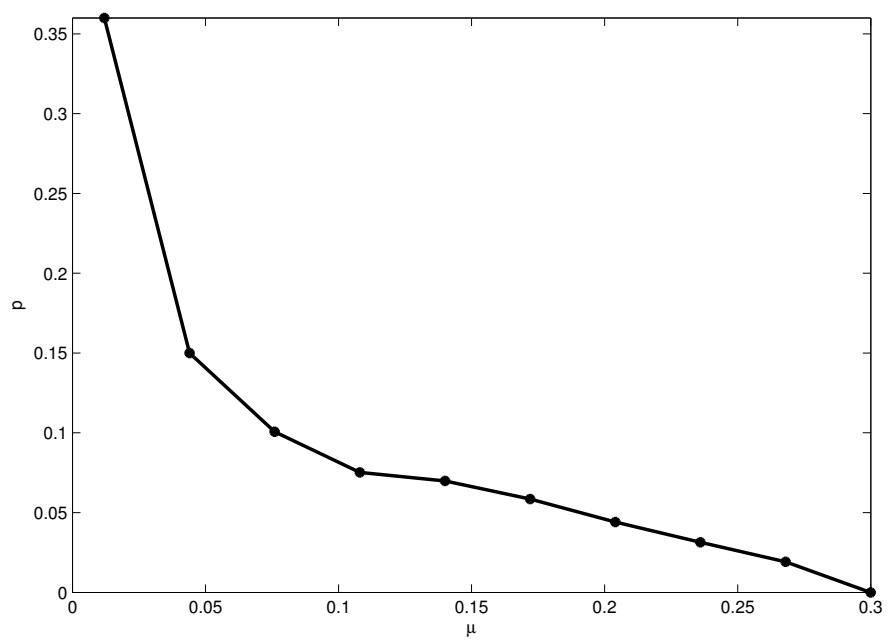

Figure 3: Indifference fee rate w.r.t. $\mu_{0}$

Notice that indifference fee rates decrease with respect to the initial drift. The bigger is the drift the less usefull are the guarantees, then the fees payed to get these guarantees have to decrease.

Figure 4 plots the indifference fee rates when the volatility $\sigma$ ranged from 0.1 to 0.4. 


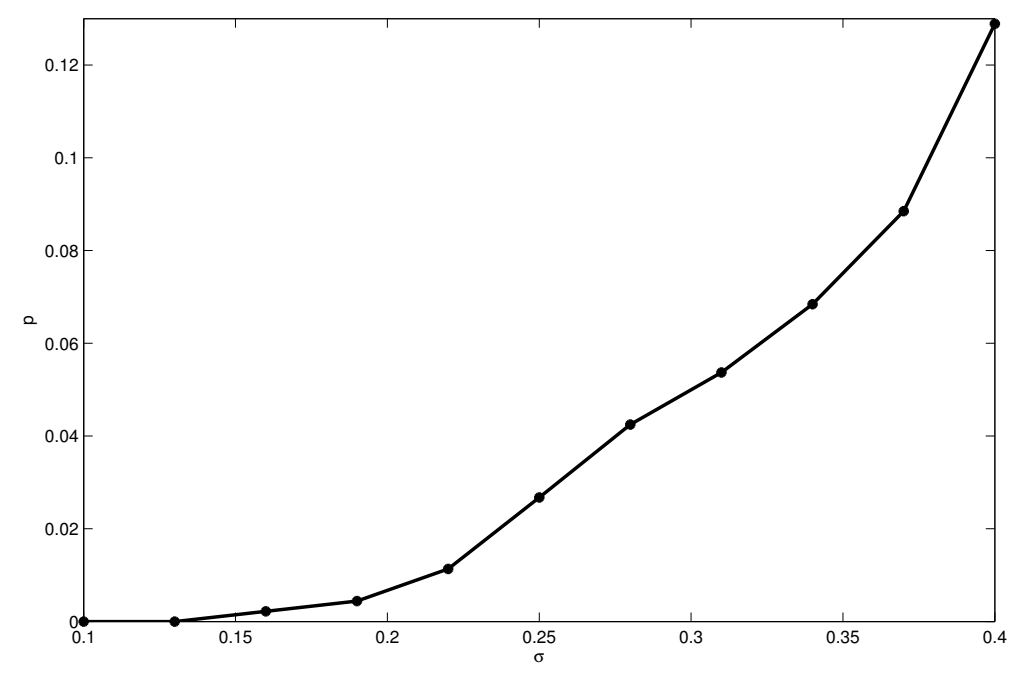

Figure 4: Indifference fee rate with respect to $\sigma$

Once again, we can get a financial interpretation of the monotonicity of the fees w.r.t. market volatility. The bigger is the volatility the more usefull are the guarantees, then the fees payed to get these guarantees have to increase.

\subsection{Longevity risk}

In this second part, we emphasize the impact of longevity risks on indifference fees for ratchet guarantees. Figure 5 plots the indifference fee rates when the intensity $\lambda$ ranged from 0 to 0.25 .

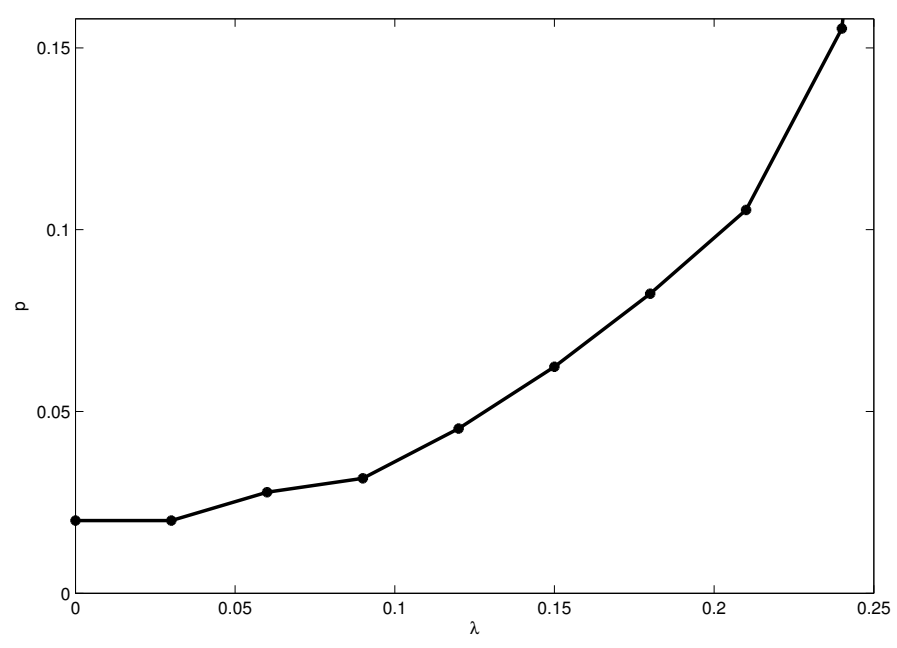

Figure 5: Indifference fee rate with respect to $\lambda$ 
Figure 6 plots the indifference fee rates when the terminal time of the contract $T$ ranged from 7 to 28 .

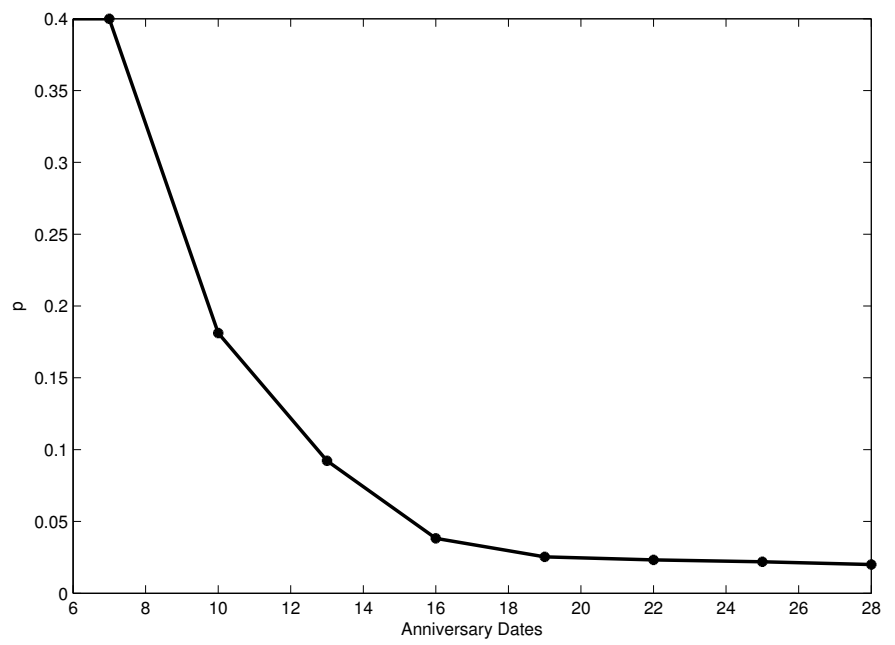

Figure 6: Indifference fee rate with respect to the maturity

Notice that the more it remains time or expected time to maturity, the more the insurer will receive fees. Hence, fee rate should decrease when time or expected time to maturity increases.

\subsection{Roll up guarantee risk}

To end this numerical section, we consider the roll-up guarantee case. We presents some sensibilities of indefference fee rates to the roll-up rate $\eta$, to the initial investment $A_{0}$ and to the withdrawal rate $\xi$.

Figure 7 plots the indifference fee rates when the roll-up rate $\eta$ ranged from 0 to 0.05 .

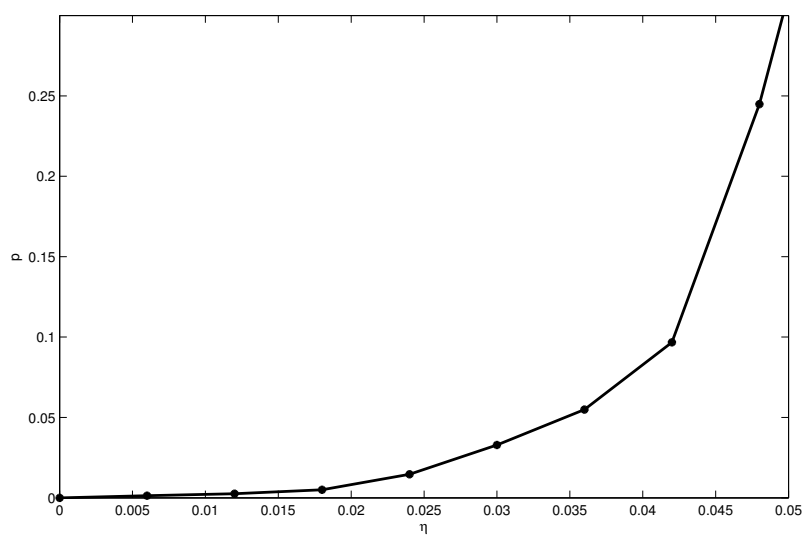

Figure 7: Indifference fee rate with respect to $\eta$

We remark that the indifference fee rates are increasing with respect to the roll-up rate 
$\eta$ with an exponential growth. The insurer has to be carefull when he offers a roll up guarantee: if he proposes a rate $\eta$ too high (for example $\eta>0.05$ ), the guarantee could be not rentable to sell, at any price.

Figure 8 plots the indifference fee rates when the initial value $A_{0}$ ranged from 0.5 to 2 .

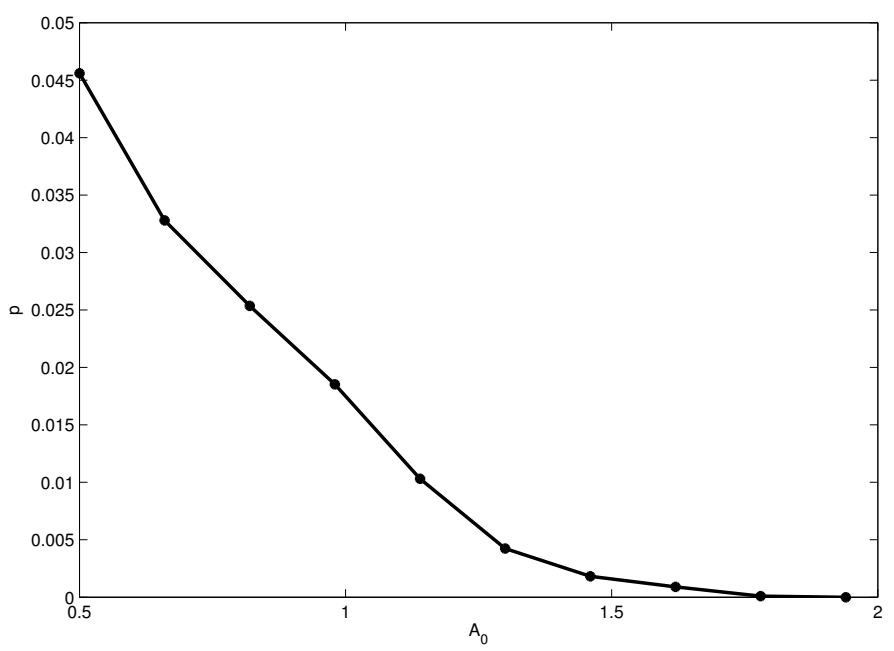

Figure 8: Indifference fee rate with respect to $A_{0}$

As expected, indifference fee rates are decreasing with respect to the initial investment $A_{0}$. If $A_{0}$ is too small it could be not interesting for the insurer to sell the product, whatever the fees are.

Figure 9 plots the indifference fee rates when the withdrawal rate $\xi$ is constant and ranged from 0 to 0.3 . It shows that indifference fee rates are lineary increasing w.r.t. the withdrawal rate $\xi$.

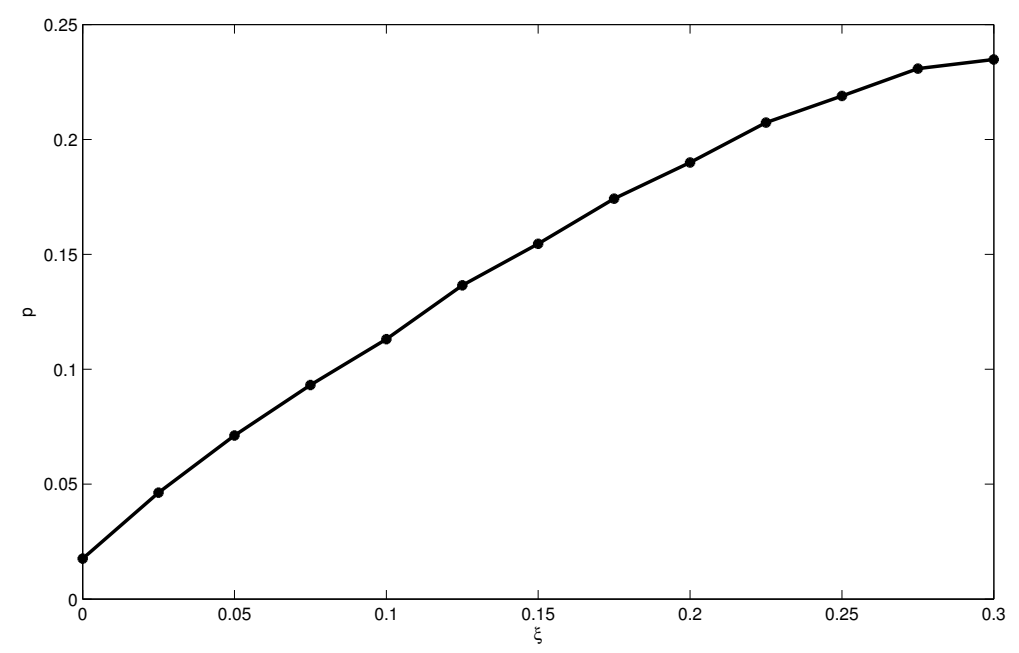

Figure 9: Indifference fee rate with respect to $\xi$ 


\section{A Appendix}

\section{A.1 Utility maximization between $T \wedge \tau$ and $T$}

Lemma A.1. There exists a strategy $\pi^{*, \tau} \in \mathcal{A}^{\mathbb{G}}[T \wedge \tau, T]$ such that

$$
\underset{\pi \in \mathcal{A}^{\mathbb{G}}[T \wedge \tau, T]}{\operatorname{ens} \inf } \mathbb{E}\left[\exp \left(-\gamma \Delta X_{\tau, T}^{\pi}\right) \mid \mathcal{G}_{T \wedge \tau}\right]=\mathbb{E}\left[\exp \left(-\gamma \Delta X_{\tau, T}^{\pi^{*, \tau}}\right) \mid \mathcal{G}_{T \wedge \tau}\right]
$$

Moreover, there exists a process $Y^{(\tau)}$ such that

$$
\underset{\pi \in \mathcal{A}^{\mathbb{G}}[T \wedge \tau, T]}{\operatorname{essinf}} \mathbb{E}\left[\exp \left(-\gamma \Delta X_{T \wedge \tau, T}^{\pi}\right) \mid \mathcal{G}_{T \wedge \tau}\right]=\exp \left(\gamma Y_{T \wedge \tau}^{(\tau)}\right)
$$

where $\left(Y^{(\tau)}, Z^{(\tau)}\right)$ is solution of the BSDE

$$
\left\{\begin{array}{l}
d Y_{t}^{(\tau)}=\left[\frac{\nu_{t}^{2}}{\gamma}+\nu_{t} Z_{t}^{(\tau)}\right] d t+Z_{t}^{(\tau)} d B_{t}, \\
Y_{T}^{(\tau)}=0
\end{array}\right.
$$

Proof. We look for a process $Y^{(\tau)}$ such that the family of processes $\left\{J^{(\tau)}(\pi), \pi \in \mathcal{A}^{\mathbb{G}}[T \wedge \tau, T]\right\}$ defined for any $\pi \in \mathcal{A}^{\mathbb{G}}[T \wedge \tau, T]$ by

$$
J_{t}^{(\tau)}(\pi):=\exp \left(-\gamma\left(\Delta X_{\tau, t}^{\pi}-Y_{t}^{(\tau)}\right)\right)
$$

satisfied the following conditions

(i) $J_{T}^{(\tau)}(\pi)=\exp \left(-\gamma \Delta X_{\tau, T}^{\pi}\right)$.

(ii) $J_{T \wedge \tau}^{(\tau)}(\pi)$ is a random variable $\mathcal{G}_{T \wedge \tau}$-measurable and independent of $\pi$.

(iii) $J^{(\tau)}(\pi)$ is a submartingale for any $\pi \in \mathcal{A}^{\mathbb{G}}[T \wedge \tau, T]$ on the time interval $[T \wedge \tau, T]$.

(iv) There exists a strategy $\pi^{*, \tau}$ such that $J^{(\tau)}\left(\pi^{*, \tau}\right)$ is a martingale on the time interval $[T \wedge \tau, T]$.

The process $Y^{(\tau)}$ is looked under the form

$$
\begin{cases}-d Y_{t}^{(\tau)} & =f\left(t, Y_{t}^{(\tau)}, Z_{t}^{(\tau)}\right) d t-Z_{t}^{(\tau)} d B_{t}, \\ Y_{T}^{(\tau)} & =0\end{cases}
$$

and we are bounded to choose the function $f$ for which $J^{(\tau)}(\pi)$ satisfies the previous conditions. Classically we obtain

$$
f(t, y, z)=-\frac{\nu_{t}^{2}}{\gamma}-\nu_{t} z
$$

and the candidate to be $\pi^{*, \tau}$ is given by

$$
\pi_{t}^{*, \tau}=\frac{1}{\hat{\sigma}_{t}}\left[\frac{\nu_{t}}{\gamma}+Z_{t}^{(\tau)}\right], \quad \forall t \in[T \wedge \tau, T] .
$$


The end of the proof is identical to the one in [15].

\section{A.2 Proof of Lemma 2.2}

We denote the upper bound of the uniformly bounded process $Y^{0}(p)$ by $k$. Applying Itï $₫ \frac{1}{2}$ 's formula to $\left(Y^{0}(p)-k\right)^{2}$, we obtain, for any $\mathbb{G}$-stopping times $\theta \leq T$,

$$
\left|Y_{T}^{0}(p)-k\right|^{2}-\left|Y_{\theta}^{0}(p)-k\right|^{2}=2 \int_{\theta}^{T}\left(Y_{s}^{0}(p)-k\right) d Y_{s}^{0}(p)+\int_{\theta}^{T}\left|Z_{s}^{0}(p)\right|^{2} d s .
$$

Taking the conditional expected value, we get

$$
\begin{aligned}
\mathbb{E}\left[\int_{\theta}^{T}\left|Z_{s}^{0}(p)\right|^{2} d s \mid \mathcal{G}_{\theta}\right]= & 2 \mathbb{E}\left[\int_{\theta}^{T}\left(k-Y_{s}^{0}(p)\right)\left[\frac{\nu_{s}^{2}}{2 \gamma}+\nu_{s} Z_{s}^{0}(p)-\lambda_{s} \frac{e^{\gamma\left(F_{s}^{D, W}(p)-Y_{s}^{0}(p)\right)}-1}{\gamma}\right] \mid \mathcal{G}_{\theta}\right] \\
& +\mathbb{E}\left[\left|F_{T}^{L}(p)-k\right|^{2} \mid \mathcal{G}_{\theta}\right]-\left|Y_{\theta}^{0}(p)-k\right|^{2} .
\end{aligned}
$$

Due to Assumption $\mathbf{A} \mathbf{1}$ and the fact that $Y^{0}(p) \in S_{\mathbb{G}}^{\infty}$, there exist two positive constants $c_{1}$ and $c_{2}$ such that

$$
\begin{aligned}
\mathbb{E}\left[\int_{\theta}^{T}\left|Z_{s}^{0}(p)\right|^{2} d s \mid \mathcal{G}_{\theta}\right] & \leq c_{1}+c_{1} \mathbb{E}\left[\int_{\theta}^{T} Z_{s}^{0}(p) d s \mid \mathcal{G}_{\theta}\right] \\
& \leq c_{1}+c_{1} \mathbb{E}\left[\int_{\theta}^{T}\left(\frac{1}{2 c_{2}}\left|Z_{s}^{0}(p)\right|^{2}+\frac{c_{2}}{2}\right) d s \mid \mathcal{G}_{\theta}\right] .
\end{aligned}
$$

Therefore, there exists a positive constant $c$ such that

$$
\mathbb{E}\left[\int_{\theta}^{T}\left|Z_{s}^{0}(p)\right|^{2} d s \mid \mathcal{G}_{\theta}\right] \leq c
$$

Hence $\int_{0}^{*} Z_{s}^{0}(p) d B_{s}$ is a $\mathrm{BMO}(\mathbb{P})$-martingale. By definition of $\pi^{*}$, Assumption $\mathbf{A} 1$ and using the results of [15] for $Z^{(\tau)}$, it follows that $\int_{0}^{*} \sigma_{s} \pi_{s}^{*} d B_{s}$ is a $\mathrm{BMO}(\mathbb{P})$-martingale, since the processes $\mu, \sigma, \gamma$ and $r$ are bounded.

\section{Acknowledgments}

The support of Chaire "Markets in transition" (FBF) and the French ANR research grant LIQUIRISK are acknowledged.

\section{References}

[1] Ankirchner S., Blanchet-Scalliet C. and A. Eyraud-Loisel (2009): "Credit risk premia and quadratic BSDEs with a single jump", International Journal of Theoretical and Applied Finance, 13 (7), 1103-1129. 
[2] Bauer D., Kling A. and J. Russ (2008): "A universal pricing framework for guaranteed minimum benefits in variable annuities", Astin Bull, 38, 621-651.

[3] Belanger A.-C., Forsyth P.-A. and G. Labahn (2009): "Valuing the Guaranteed Minimum Death Benefit Clause with Partial Withdrawals", Applied Mathematical Finance, 16 (6), 451-496.

[4] Bielecki T., Jeanblanc M. and M. Rutkowski (2004): "Stochastic Methods in Credit Risk Modelling", Lectures notes in Mathematics, Springer, 1856, 27-128.

[5] Borch K. (1990) "Economics of insurance", Amsterdam: Elsevier Science Publishers B. $V$..

[6] Briand P., J.-P. Lepeltier and J. San Martin (2007): "One-dimensional backward stochastic differential equations whose coefficient is monotonic in $y$ and non-Lipschitz in $z "$, Bernouilli, 13 (1), 80-91.

[7] Bouchard B. and N. Touzi (2004) "Discrete-time approximation and Monte-Carlo simulation of backward stochastic differential equations", Stochastic Processes and their Applications, 111 (2), 175-206.

[8] Boyle P.-P. and E. Schwartz (1977): "Equilibrium prices of guarantees under equitylinked contracts", Journal of Risk and Insurance, 44 (2), 639-680.

[9] Carmona R. (2009). "Indifference Pricing: Theory and Applications", Princeton University Press.

[10] Chu C.-C. and Y.-K. Kwok (2004): "Reset and withdrawal rights in dynamic fund protection", Insurance: Mathematics and Economics, 34, 273-295.

[11] Dai M., Kwok Y.-K. and J. Zong (2008): "Guaranted minimum withdrawal benefit in variable annuities", Mathematical Finance, 34, 273-295.

[12] El Karoui N., Peng S. and M.-C. Quenez (1997): "Backward Stochastic Differential Equations in Finance", Mathematical Finance, 1-71.

[13] El Karoui N., Kapoudjian C., Pardoux E., Peng S. and M.-C. Quenez (1997): "Reflected solutions of backward SDE's, and related obstacle problems for PDE's", Annals of Probabilty, 2, 702-737.

[14] Fan S.-J. and L. Jiang (2010): "Uniqueness result for the BSDE whose generator is monotonic in $y$ and uniformly continuous in $z "$, Les Comptes Rendues de l'Acadi $; \frac{1}{2}$ mie des sciences de Paris, 348, 89-92.

[15] Hu Y., Imkeller P. and M. Muller (2005): "Utility maximization in incomplete markets", Annals of Probability, 15, 1691-1712.

[16] Kazamaki N. (1994): "Continuous martingales and BMO", Lectures Notes, 1579, Springer-Verlag. 
[17] Kharroubi I., Lim T. and A. Ngoupeyou (2013): "Mean-variance hedging on uncertain time horizon in a market with a jump", forthcoming in Applied Mathematics and Optimization.

[18] Milevsky M. and S. Posner (2001): "The titanic option: valuation of the guaranteed minimum death benefit in variable annuities and mutual funds", The Journal of Risk and Insurance, 68, 93-128.

[19] Milevsky M. and T.-S. Salisbury (2006): "Financial valuation of guaranteed minimum withdrawal benefits", Insurance: Mathematics and Economics, 38 (1), 21-38.

[20] Rouge R. and N. El Karoui (2000): "Pricing via utility maximization and entropy", Mathematical Finance, 10 (2), 259-276.

[21] Siu T.-K. (2005): "Fair valuation of participating policies with surrender options and regime switching", Insurance: Mathematics and Economics, 37 (3), 533-552.

[22] Sloane W.-R. (1971): "Life insurers, Variable Annuities and Mutual Fund: a Critical Study", Journal of Risk and Insurance, 38 (3), 483-486. 